\title{
Human Capital Impacts of Income Inequality: An Extensive Empirical Analysis From The African Continent
}

\section{Swapnanil Sengupta ( $\square$ swapnanil.sengupta@student.uni-tuebingen.de)}

Eberhard Karls University of Tübingen

\section{Research}

Keywords: Income inequality, Life expectancy at birth, Human capital formation, Africa, IV-2SLS, PECM

Posted Date: October 12th, 2021

DOI: https://doi.org/10.21203/rs.3.rs-961606/v1

License: (c) (i) This work is licensed under a Creative Commons Attribution 4.0 International License. Read Full License 


\section{Abstract}

This paper evaluates the impacts of income inequality on life expectancy in the African countries. The empirical analysis has been performed on a panel dataset of 52 African nations covering the period of 1995-2018. For estimating the relationship, I have employed Two-Stage Least Squares (2SLS) technique and a Panel Error Correction Model (PECM). The long-run cointegrating relationship was estimated using a Panel Dynamic Ordinary Least Square (PDOLS) estimator. The outputs of both static and dynamic estimation models suggest that income inequality has negatively affected life expectancy at birth in the African continent overall. Though a positive short-run causal relationship was established, in the long-run, income inequality had deleterious effects. A series of steps had been followed to check the soundness of the result of the main empirical examination and it was confirmed that the results are robust.

\section{Introduction}

\section{"A nation will not survive morally or economically when so few have so much and so many have so little"}

-Bernie Sanders

(US Senator)

In 2011, the World Economic Forum established that the income inequality and corruption are the two most serious challenges in front of the world (World Economic Forum, 2011). The World Social Report 2020, published by UNDESA asserts that the inequality is rising for more than 70 percent of the global population, thwarting socio-economic development. It has been well over four decades since Rodgers (1979) reported the associations of health outcomes and income inequality, in his seminal work. His work, along with Wilkinson's $(1992,1996)$, gained traction and inspired further research on the subject matter in the following decades (for eg., Pickett \& Wilkinson, 2015; Truesdale \& Jencks, 2016). Apparently, one of the several channels via which income inequality proves to be detrimental to the economy is via the health facet of human capital. As mentioned by Wilkinson (1996), the distribution of income is "one of the most powerful influences on the health of whole population in the developed world to have come to light". Two primary hypotheses prevail to illustrate the ecological association between population health and income inequality: the absolute income hypothesis and the contextual inequality hypothesis, also known as the "Wilkinson Hypothesis". The absolute income hypothesis maintains that the uneven income distribution does not directly affect individual health. Instead, the association between income inequality and population health is purely an outcome of a curvilinear relation between income and individual health, i.e., a diminishing return of health to income (Deaton, 2003; Gravelle, 1998). The argument is depicted in Figure 1. Assume that health is nonlinear concave function of income, such that an increase in income causes diminishing returns of health and, that a hypothetical economy consists of only two equal-sized cohorts, the rich with income $x_{4}$ and the poor with income $x_{1}$. The average population health is $y_{1}$. If an amount of money $\left(x_{4}-x_{1}\right)$ is taken away from the rich and given to the poor, the poor's health will improve and the rich's health will deteriorate. Due to diminishing health return to income, the poor will have gained more health than the rich would have lost. Therefore, the population health increases from $y_{1}$ to $y_{2}$, as income inequality decreases, though the average income in the society remains unchanged.

On the other side, the Wilkinson hypothesis advances a direct, contextual and causal income inequality impacts on the health of individuals, over and above underlying individual-level socio-economic determinants of health, inclusive of individual's income (Wilkinson, 1992, 1996, 2001). Income inequality stands as a hindrance in the path of nutrition consumption and accessing healthcare services due to disproportionate income emanating in poor households (Birdsall et al., 1995). Health conditions become aggravated in the case of income inequality even beyond the absolute poverty (or, read income) due to the psychological consequences of social and status comparisons, rise in violent 
crimes and murders (Pickett, Mookherjee, et al., 2005), increased levels of obesity (Pickett, Kelly, et al., 2005), higher levels of infant mortality (Spencer, 2004) and bad self-reported health after adjusting for poverty levels and societal wealth, which in turn, reduces life expectancy in both developed and developing economies (Babones, 2008; Hales et al., 1999; Pei \& Rodriguez, 2006; Rasella et al., 2013; S. V. Subramanian et al., 2003). It is posited that disparities in income distribution deleteriously impacts health outcomes of both the poor and rich via the psychosocial, structural, social capital or neo-material pathways (Aida et al., 2011; Elgar, 2010; Kawachi \& Kennedy, 1997; Layte, 2012; Lynch et al., 2000; Mellor \& Milyo, 2002; Pickett \& Wilkinson, 2015; Ribeiro et al., 2017; Rözer \& Volker, 2015; S. V. Subramanian \& Kawachi, 2004; Torre \& Myrskylä, 2011; Wilkinson, 1996, 1999). Psychosocial factors can be explained as the feeling of relative deprivation due to broadened gaps in financial conditions which results in frustration, stress, shame, and anxiety, which in turn, induces self-sabotaging behaviours like smoking and alcohol consumption (De Botton, 2004; Eibner \& Evans, 2005; Wilkinson, 2001). Disproportionate income distribution might reduce social capital as well. Elgar (2010) discovered that income inequality was strongly associated to interpersonal trust, and that reduced interpersonal trust was linked to higher adult mortality, as well as diminished life expectancy. It has also been found that societies that condone higher levels of income inequality, tend to underinvest in wide array of physical, human, health and social infrastructures that improves population health (Detollenaere et al., 2018; Kaplan et al., 1996; Pearce \& Smith, 2003; Rostila et al., 2012; G. D. Smith, 1996). Krugman (1996) and Deaton (2003) shed light on why higher income inequality causes lower social spending. They explained that uneven income distribution intensifies the divergence in interests between cohorts of different financial conditions.

However, the causal effects of income inequality on health are not unanimously agreed on. The proponents of the "Income Inequality Hypothesis" proclaimed that it was the income distribution rather than the absolute income that governed the health consequences (Runciman, 1966; Wilkinson, 1996). In fact, a lot of studies support the claim (for e.g., Le Grand, 1987; Rasella et al., 2013; Rodgers, 1979). On the opposing end, doubts have been cast on the robustness of this relationship. The reason cited was that if the individual health is a non-linear function of income, then income inequality might be spuriously correlated with aggregate health measures (Gravelle et al., 2002; Judge et al., 1998). Mellor and Milyo (2002) too made their scepticism clear by arguing that it is necessary to use individual-level data and individual income in the regression models to have a clear picture of the causal effect of income inequality on health. Many researchers did not find any link between the two variables as well (for e.g., Grönqvist et al., 2012; Judge, 1995; Wen et al., 2003).

Not losing sight of all these facts, Africa continues to be afflicted by overwhelmingly uneven income and wealth distributions, in spite of the impressive economic progress in the recent decades. In fact, a new UNDP report found a staggering figure that 10 of the world's 19 most unequal countries are in sub-Saharan Africa. However, very little to no attention has been given on examining the impacts of income inequality on the health aspect of the human capital. This paper aims to empirically analyse the health impacts of income inequality in the entirety of the African continent, extensively. The empirical analysis has been performed on a panel dataset of 52 African nations covering the period of 1995-2018, inclusive. Life expectancy at birth has been used as the proxy for health conditions. For estimating the relationship, IV-2SLS technique and a Panel Error Correction Model (PECM) have been employed. The instrument used for inequality in the IV-2SLS estimation is agricultural land per capita and the long-run cointegrating relationship is estimated using a Panel Dynamic Ordinary Least Square (PDOLS) estimator. This study contributes to the existing literature in the following ways: i) it covered the entire African continent, ii) used a large dataset covering a longer period, iii) introduced a new instrument for inequality, iv) employed never used estimation techniques for this particular subject matter in the concerned location, and v) provided a detailed and up-to-date literature review.

The rest of the paper is structured as follows: Section 2 reviews the existing literature; Section 3 presents the empirical analysis to shed light on the theory, and Section 4 draws the conclusion of the study. 
[1] The hypothesis has been explained with reference to (Luo \& Xie, 2020)

\section{Literature Review}

A multitude of empirical researches have been conducted since forever, to understand the income inequality-health hypothesis and consequently, shed light on how income distributions impact the human capital from health perspective, across countries. It can be safely asserted that, just like the takes on the subject matter by different economists are not unanimous, the empirical findings are not uniform as well. A broad spectrum of findings exists, with most conforming to the negative impacts of income inequality on health, some find no relationships and some has conclusions exactly on the opposite end. For convenience in analysing the existing studies, the review has been presented in the tabular format below: 
Table 1

Empirical Studies on Health Impacts of Income Inequality

\begin{tabular}{|c|c|c|c|c|c|c|}
\hline $\mathrm{S} / \mathrm{N}$ & $\begin{array}{l}\text { Author(s), } \\
\text { Year }\end{array}$ & Sample & $\begin{array}{l}\text { Estimation } \\
\text { Method }\end{array}$ & $\begin{array}{l}\text { Proxy(s) } \\
\text { of Income } \\
\text { Inequality }\end{array}$ & $\begin{array}{l}\text { Proxy(s) of } \\
\text { Health }\end{array}$ & Findings \\
\hline 1. & $\begin{array}{l}\text { Rodgers } \\
\text { (1979) }\end{array}$ & $\begin{array}{l}56 \text { countries } \\
\text { (DCs \& LDCs) }\end{array}$ & $\begin{array}{l}\text { Multiple } \\
\text { Regression }\end{array}$ & $\begin{array}{l}\text { GC and } \\
\text { others }\end{array}$ & LE and IMR & $\begin{array}{l}\text { Higher income inequality } \\
\text { strongly associated to } \\
\text { lower life expectancy }\end{array}$ \\
\hline 2. & Flegg (1979) & $\begin{array}{l}60 \text { non- } \\
\text { industrialised } \\
\text { countries }\end{array}$ & OLS & $\begin{array}{l}\text { Atkinson } \\
\text { Index }\end{array}$ & Fertility Rate & $\begin{array}{l}10 \% \text { reduction in } \\
\text { Atkinson index leads to } \\
\text { reduced birth rates by } \\
2.2 \%\end{array}$ \\
\hline 3. & Flegg (1982) & $\begin{array}{l}46 \text { LDCs and } \\
13 \text { DCs }\end{array}$ & OLS; 2SLS & Gini Index & IMR & Inequality increases IMR \\
\hline 4. & $\begin{array}{l}\text { Pampel and } \\
\text { Pillai (1986) }\end{array}$ & $\begin{array}{l}18 \\
\text { industrialised } \\
\text { countries }\end{array}$ & OLS & Gini Index & IMR & $\begin{array}{l}\text { No association between } \\
\text { higher income inequality } \\
\text { and IMR }\end{array}$ \\
\hline 5. & $\begin{array}{l}\text { Wilkinson } \\
\text { (1992) }\end{array}$ & $\begin{array}{l}9 \\
\text { industrialised } \\
\text { countries }\end{array}$ & $\begin{array}{l}\text { Multiple } \\
\text { Regression }\end{array}$ & $\begin{array}{l}\text { Proportion } \\
\text { of income } \\
\text { to those } \\
\text { below } 7 \text { th } \\
\text { decile }\end{array}$ & LE & $\begin{array}{l}\text { Higher income inequality } \\
\text { strongly correlated to } \\
\text { reduced life expectancy }\end{array}$ \\
\hline 6. & $\begin{array}{l}\text { Wennemo } \\
\text { (1993) }\end{array}$ & $\begin{array}{l}11 \\
\text { industrialised } \\
\text { countries }\end{array}$ & $\begin{array}{l}\text { Multiple } \\
\text { Regression }\end{array}$ & Gini Index & IMR & $\begin{array}{l}\text { Higher income inequality } \\
\text { associated to higher } \\
\text { infant mortality rate }\end{array}$ \\
\hline 7. & Judge (1995) & $\begin{array}{l}13 \\
\text { industrialised } \\
\text { countries }\end{array}$ & $\begin{array}{l}\text { Multiple } \\
\text { Regression }\end{array}$ & $\begin{array}{l}\text { Gini Index; } \\
\text { income } \\
\text { shares }\end{array}$ & LE & $\begin{array}{l}\text { No relation between } \\
\text { income inequality and LE }\end{array}$ \\
\hline 8. & $\begin{array}{l}\text { Kawachi et } \\
\text { al. (1997) }\end{array}$ & 39 US States & OLS & $\begin{array}{l}\text { Robin } \\
\text { Hood } \\
\text { Index; } \\
\text { Income } \\
\text { decile }\end{array}$ & AAMR & $\begin{array}{l}\text { Higher income inequality } \\
\text { correlated with higher } \\
\text { AAMR }\end{array}$ \\
\hline $\mathrm{S} / \mathrm{N}$ & $\begin{array}{l}\text { Author(s), } \\
\text { Year }\end{array}$ & Sample & $\begin{array}{l}\text { Estimation } \\
\text { Method }\end{array}$ & $\begin{array}{l}\text { Proxy(s) } \\
\text { of Income } \\
\text { Inequality }\end{array}$ & $\begin{array}{l}\text { Proxy(s) of } \\
\text { Health }\end{array}$ & Findings \\
\hline 9. & $\begin{array}{l}\text { Judge et al. } \\
\text { (1998) }\end{array}$ & $\begin{array}{l}16 \\
\text { industrialised } \\
\text { countries }\end{array}$ & OLS & $\begin{array}{l}\text { Income } \\
\text { Shares, GI }\end{array}$ & LE, IMR & $\begin{array}{l}\text { No relation between } \\
\text { inequality and LE; no } \\
\text { relation between change } \\
\text { in inequality and change } \\
\text { in LE and IMR }\end{array}$ \\
\hline
\end{tabular}

Note: $\mathrm{AAMR} \Rightarrow$ Age Adjusted Mortality Rate; $\mathrm{ADL} \Rightarrow$ Activities of Daily Living; $\mathrm{BMI} \Rightarrow$ Body Mass Index; $\mathrm{CHC} \Rightarrow$ Chronic Health Conditions; CSMR $\Rightarrow$ Cause-Specific Mortality Rate; DCs $\Rightarrow$ Developed Countries; GI $\Rightarrow$ Gini Index; $\mathrm{HUI} \Rightarrow$ Health Utility Index; IMR $\Rightarrow$ Infant Mortality Rate; IRHI $\Rightarrow$ Income-Related Health Inequality; LDCs $\Rightarrow$ Less Developed Countries; LE $\Rightarrow$ Life Expectancy; MAMC $\Rightarrow$ Middle-upper Arm Muscle Circumference; SRH $\Rightarrow$ SelfRated Health; TB $\Rightarrow$ Tuberculosis; UMR $\Rightarrow$ Under-5 Mortality Rate; WHR $\Rightarrow$ Waist-to-Hip Ratio; WHZ $\Rightarrow$ Weight for Height-Z-score 


\begin{tabular}{|c|c|c|c|c|c|c|}
\hline$S / N$ & $\begin{array}{l}\text { Author(s), } \\
\text { Year }\end{array}$ & Sample & $\begin{array}{l}\text { Estimation } \\
\text { Method }\end{array}$ & $\begin{array}{l}\text { Proxy(s) } \\
\text { of Income } \\
\text { Inequality }\end{array}$ & $\begin{array}{l}\text { Proxy(s) of } \\
\text { Health }\end{array}$ & Findings \\
\hline 10. & $\begin{array}{l}\text { Walberg et al. } \\
(1998)\end{array}$ & Russia & $\begin{array}{l}\text { Probit } \\
\text { Regression }\end{array}$ & $\begin{array}{l}\text { Robin } \\
\text { Hood } \\
\text { index }\end{array}$ & Change in LE & $\begin{array}{l}\text { Higher income inequality } \\
\text { leads to lower life } \\
\text { expectancy }\end{array}$ \\
\hline 11. & $\begin{array}{l}\text { Chiang } \\
\text { (1999) }\end{array}$ & Taiwan & OLS & $\begin{array}{l}50 \% \\
\text { Income } \\
\text { Shares, GI }\end{array}$ & AAMR, UMR & $\begin{array}{l}\text { Weak association } \\
\text { between income } \\
\text { inequality and AAMR but } \\
\text { UMR associated with } \\
\text { higher income inequality }\end{array}$ \\
\hline 12. & $\begin{array}{l}\text { Wolfson et } \\
\text { al. (1999) }\end{array}$ & 50 US States & $\begin{array}{l}\text { Multiple } \\
\text { regression }\end{array}$ & Gini Index & $\begin{array}{l}\text { Risk of } \\
\text { mortality }\end{array}$ & $\begin{array}{l}\text { Weaker link between the } \\
\text { level of income inequality } \\
\text { and observed mortality in } \\
\text { each state }\end{array}$ \\
\hline 13. & $\begin{array}{l}\text { LeClere and } \\
\text { Soobadar } \\
(2000)\end{array}$ & US counties & $\begin{array}{l}\text { Logistic } \\
\text { Regression }\end{array}$ & Gini Index & SRH & $\begin{array}{l}\text { Higher income inequality } \\
\text { correlated with poorer } \\
\text { SRH, for only whites with } \\
\text { age } 18 \text { to } 44 \text { and not } \\
\text { associated in other age } \\
\text { groups }\end{array}$ \\
\hline 14. & $\begin{array}{l}\text { Mellor and } \\
\text { Milyo (2002) }\end{array}$ & 50 US States & $\begin{array}{l}\text { Probit } \\
\text { Regression }\end{array}$ & Gini Index & SRH & $\begin{array}{l}\text { No relation between } \\
\text { income inequality and } \\
\text { SRH }\end{array}$ \\
\hline 15. & $\begin{array}{l}\text { Shibuya et al. } \\
(2002)\end{array}$ & Japan & $\begin{array}{l}\text { Probit } \\
\text { Regression }\end{array}$ & Gini Index & SRH & $\begin{array}{l}\text { Higher income inequality } \\
\text { not associated with an } \\
\text { increased likelihood of } \\
\text { poor health }\end{array}$ \\
\hline 16. & $\begin{array}{l}\text { Shi et al. } \\
\text { (2003) }\end{array}$ & US States & $\begin{array}{l}\text { Cross- } \\
\text { sectional } \\
\text { analysis }\end{array}$ & Gini Index & $\begin{array}{l}\text { Stroke } \\
\text { mortality }\end{array}$ & $\begin{array}{l}\text { Income inequality has a } \\
\text { reduced effect on stroke } \\
\text { mortality }\end{array}$ \\
\hline 17. & $\begin{array}{l}\text { Wen et al. } \\
\text { (2003) }\end{array}$ & US States & $\begin{array}{l}\text { Hierarchical } \\
\text { ordinal logit } \\
\text { model }\end{array}$ & Gini Index & SRH & $\begin{array}{l}\text { Income inequality is not a } \\
\text { structurally important } \\
\text { determinant of SRH }\end{array}$ \\
\hline$S / N$ & $\begin{array}{l}\text { Author(s), } \\
\text { Year }\end{array}$ & Sample & $\begin{array}{l}\text { Estimation } \\
\text { Method }\end{array}$ & $\begin{array}{l}\text { Proxy(s) } \\
\text { of Income } \\
\text { Inequality }\end{array}$ & $\begin{array}{l}\text { Proxy(s) of } \\
\text { Health }\end{array}$ & Findings \\
\hline 18. & $\begin{array}{l}\text { Messias } \\
\text { (2003) }\end{array}$ & $\begin{array}{l}\text { Brazilian } \\
\text { States }\end{array}$ & $\begin{array}{l}\text { Simple and } \\
\text { Multiple } \\
\text { Linear } \\
\text { Regression }\end{array}$ & Gini Index & LE & $\begin{array}{l}\text { Income inequality } \\
\text { negatively affects LE }\end{array}$ \\
\hline
\end{tabular}

Note: $\mathrm{AAMR} \Rightarrow$ Age Adjusted Mortality Rate; $\mathrm{ADL} \Rightarrow$ Activities of Daily Living; $\mathrm{BMI} \Rightarrow$ Body Mass Index; $\mathrm{CHC} \Rightarrow$ Chronic Health Conditions; CSMR $\Rightarrow$ Cause-Specific Mortality Rate; DCs $\Rightarrow$ Developed Countries; GI $\Rightarrow$ Gini Index; $\mathrm{HUI} \Rightarrow$ Health Utility Index; IMR $\Rightarrow$ Infant Mortality Rate; IRHI $\Rightarrow$ Income-Related Health Inequality; LDCs $\Rightarrow$ Less Developed Countries; LE $\Rightarrow$ Life Expectancy; MAMC $\Rightarrow$ Middle-upper Arm Muscle Circumference; SRH $\Rightarrow$ SelfRated Health; TB $\Rightarrow$ Tuberculosis; UMR $\Rightarrow$ Under-5 Mortality Rate; WHR $\Rightarrow$ Waist-to-Hip Ratio; WHZ $\Rightarrow$ Weight for Height-Z-score 


\begin{tabular}{|c|c|c|c|c|c|c|}
\hline $\mathrm{S} / \mathrm{N}$ & $\begin{array}{l}\text { Author(s), } \\
\text { Year }\end{array}$ & Sample & $\begin{array}{l}\text { Estimation } \\
\text { Method }\end{array}$ & $\begin{array}{l}\text { Proxy(s) } \\
\text { of Income } \\
\text { Inequality }\end{array}$ & $\begin{array}{l}\text { Proxy(s) of } \\
\text { Health }\end{array}$ & Findings \\
\hline 19. & $\begin{array}{l}\text { McLeod et al. } \\
(2003)\end{array}$ & Canada & $\begin{array}{l}\text { Maximum } \\
\text { likelihood } \\
\text { ordered logit } \\
\text { model }\end{array}$ & $\begin{array}{l}\text { Income } \\
\text { share of } \\
\text { the } \\
\text { poorest } \\
50 \%\end{array}$ & SRH & $\begin{array}{l}\text { No association between } \\
\text { income inequality and } \\
\text { poor health }\end{array}$ \\
\hline 20. & $\begin{array}{l}\text { Subramanian } \\
\text { and Kawachi } \\
(2004)\end{array}$ & 50 US States & Logit model & Gini Index & $\mathrm{SRH}$ & $\begin{array}{l}\text { For every } 5 \% \text { increase in } \\
\text { income inequality, odds } \\
\text { ratio of having poor } \\
\text { health increased by } \\
1.32 \%\end{array}$ \\
\hline 21. & $\begin{array}{l}\text { Vogli et al. } \\
(2005)\end{array}$ & $\begin{array}{l}\text { Italy and } 25 \\
\text { DCs }\end{array}$ & $\begin{array}{l}\text { Linear } \\
\text { Regression }\end{array}$ & Gini Index & LE & $\begin{array}{l}\text { Income inequality } \\
\text { independently associated } \\
\text { with life expectancy in } \\
\text { Italy. Strong negative } \\
\text { correlation in cross } \\
\text { national analysis }\end{array}$ \\
\hline 22. & Ram (2005) & US States & OLS & Gini Index & $\begin{array}{l}\text { Death crude } \\
\text { rate }\end{array}$ & $\begin{array}{l}\text { Income inequality exerted } \\
\text { on mortality, significantly }\end{array}$ \\
\hline 23. & Ram (2006) & $\begin{array}{l}108 \text { countries } \\
\text { (cross- } \\
\text { sectional) }\end{array}$ & OLS & $\begin{array}{l}\text { Gl, income } \\
\text { share of } \\
\text { top 10\% }\end{array}$ & IMR & $\begin{array}{l}\text { Cross-country } \\
\text { association found } \\
\text { negative between income } \\
\text { inequality and health }\end{array}$ \\
\hline 24. & $\begin{array}{l}\text { Li and Zhu } \\
(2006)\end{array}$ & $\begin{array}{l}8 \text { Chinese } \\
\text { Provinces }\end{array}$ & $\begin{array}{l}\text { Probit } \\
\text { Regression }\end{array}$ & Gini Index & $\begin{array}{l}\text { SRH, physical } \\
\text { functions, } \\
\text { ADL }\end{array}$ & $\begin{array}{l}\text { Inverted U-association } \\
\text { between income } \\
\text { inequality and SRH, } \\
\text { implying higher inequality } \\
\text { poses health threats }\end{array}$ \\
\hline 25. & $\begin{array}{l}\text { Wilkinson } \\
\text { and Pickett } \\
(2006)\end{array}$ & $\begin{array}{l}\text { Countrywide } \\
\text { studies }\end{array}$ & $\begin{array}{l}\text { Meta- } \\
\text { analysis of } \\
155 \text { studies }\end{array}$ & - & - & $\begin{array}{l}\text { Negative effect of income } \\
\text { inequality on health was } \\
\text { found in } 70 \text { percent of the } \\
\text { studies }\end{array}$ \\
\hline$S / N$ & $\begin{array}{l}\text { Author(s), } \\
\text { Year }\end{array}$ & Sample & $\begin{array}{l}\text { Estimation } \\
\text { Method }\end{array}$ & $\begin{array}{l}\text { Proxy(s) } \\
\text { of Income } \\
\text { Inequality }\end{array}$ & $\begin{array}{l}\text { Proxy(s) of } \\
\text { Health }\end{array}$ & Findings \\
\hline 26. & $\begin{array}{l}\text { Subramanian } \\
\text { et al. (2007) }\end{array}$ & $\begin{array}{l}\text { India (1998- } \\
1999 \text { cross- } \\
\text { sectional } \\
\text { National } \\
\text { Family Health } \\
\text { Survey) }\end{array}$ & $\begin{array}{l}\text { Multi- } \\
\text { nominal } \\
\text { logistic } \\
\text { regression }\end{array}$ & Gini Index & $\mathrm{BMI}$ & $\begin{array}{l}\text { Risk of under and over } \\
\text { nutrition at the individual } \\
\text { level significantly } \\
\text { associated with income } \\
\text { inequality }\end{array}$ \\
\hline
\end{tabular}

Note: $\mathrm{AAMR} \Rightarrow$ Age Adjusted Mortality Rate; $\mathrm{ADL} \Rightarrow$ Activities of Daily Living; $\mathrm{BMI} \Rightarrow$ Body Mass Index; $\mathrm{CHC} \Rightarrow$ Chronic Health Conditions; CSMR $\Rightarrow$ Cause-Specific Mortality Rate; DCs $\Rightarrow$ Developed Countries; GI $\Rightarrow$ Gini Index; $\mathrm{HUI} \Rightarrow$ Health Utility Index; IMR $\Rightarrow$ Infant Mortality Rate; IRHI $\Rightarrow$ Income-Related Health Inequality; LDCs $\Rightarrow$ Less Developed Countries; LE $\Rightarrow$ Life Expectancy; MAMC $\Rightarrow$ Middle-upper Arm Muscle Circumference; SRH $\Rightarrow$ SelfRated Health; TB $\Rightarrow$ Tuberculosis; UMR $\Rightarrow$ Under-5 Mortality Rate; WHR $\Rightarrow$ Waist-to-Hip Ratio; WHZ $\Rightarrow$ Weight for Height-Z-score 


\begin{tabular}{|c|c|c|c|c|c|c|}
\hline$S / N$ & $\begin{array}{l}\text { Author(s), } \\
\text { Year }\end{array}$ & Sample & $\begin{array}{l}\text { Estimation } \\
\text { Method }\end{array}$ & $\begin{array}{l}\text { Proxy(s) } \\
\text { of Income } \\
\text { Inequality }\end{array}$ & $\begin{array}{l}\text { Proxy(s) of } \\
\text { Health }\end{array}$ & Findings \\
\hline 27. & $\begin{array}{l}\text { Safaei } \\
(2007)\end{array}$ & $\begin{array}{l}10 \text { Canadian } \\
\text { Provinces }\end{array}$ & - & $\begin{array}{l}\text { Income } \\
\text { groups }\end{array}$ & $\begin{array}{l}\text { HUl, CHC, } \\
\text { SAlH }\end{array}$ & $\begin{array}{l}\text { Income inequalities } \\
\text { caused health inequality }\end{array}$ \\
\hline 28. & $\begin{array}{l}\text { Chen and } \\
\text { Meltzer } \\
(2008)\end{array}$ & $\begin{array}{l}9 \text { Chinese } \\
\text { Provinces }\end{array}$ & $\begin{array}{l}\text { Multi-level } \\
\text { linear } \\
\text { probability } \\
\text { model }\end{array}$ & $\begin{array}{l}\text { Coefficient } \\
\text { of } \\
\text { variation }\end{array}$ & $\begin{array}{l}\text { Health risk } \\
\text { factors, } \\
\text { obesity \& } \\
\text { hypertension }\end{array}$ & $\begin{array}{l}\text { Health risk factors } \\
\text { positively associated with } \\
\text { increasing community } \\
\text { income inequality }\end{array}$ \\
\hline 29. & $\begin{array}{l}\text { Babones } \\
(2008)\end{array}$ & $\begin{array}{l}126 \text { countries } \\
(1970 \text { and } \\
1995)\end{array}$ & $\begin{array}{l}\text { Polynomial } \\
\text { Regression }\end{array}$ & Gini Index & $\begin{array}{l}\text { LE, IMR and } \\
\text { Murder rate }\end{array}$ & $\begin{array}{l}\text { Income inequality } \\
\text { significantly correlated } \\
\text { with LE, IMR and } \\
\text { inconsistently with } \\
\text { murder rate }\end{array}$ \\
\hline 30. & $\begin{array}{l}\text { Kondo et al. } \\
(2009)\end{array}$ & 11 countries & $\begin{array}{l}\text { Meta } \\
\text { regression }\end{array}$ & Gini Index & $\begin{array}{l}\text { Premature } \\
\text { mortality and } \\
\text { SRH }\end{array}$ & $\begin{array}{l}\text { Income inequality is } \\
\text { linked with a modest } \\
\text { excess risk of premature } \\
\text { mortality and SRH }\end{array}$ \\
\hline 31. & $\begin{array}{l}\text { Biggs et al. } \\
(2010)\end{array}$ & $\begin{array}{l}22 \text { Latin } \\
\text { American } \\
\text { countries } \\
(1960-2007)\end{array}$ & $\begin{array}{l}\text { Fixed } \\
\text { Effects }\end{array}$ & Gini Index & $\begin{array}{l}\text { LE, IMR and } \\
\text { TB }\end{array}$ & $\begin{array}{l}\text { Inequality has no } \\
\text { significant direct effect } \\
\text { on public health }\end{array}$ \\
\hline 32. & $\begin{array}{l}\text { Torre and } \\
\text { Myrskylä } \\
(2011)\end{array}$ & $\begin{array}{l}21 \text { rich } \\
\text { countries } \\
(1975-2006)\end{array}$ & $\begin{array}{l}\text { Random } \\
\text { Effects }\end{array}$ & Gini Index & ASMR and LE & $\begin{array}{l}\text { Strong positive } \\
\text { association between } \\
\text { income inequality and } \\
\text { mortality rate }\end{array}$ \\
\hline 33. & $\begin{array}{l}\text { Esmaeili et } \\
\text { al. (2011) }\end{array}$ & $\begin{array}{l}24 \text { Islamic } \\
\text { countries } \\
(1996-2004)\end{array}$ & OLS & $\begin{array}{l}\text { Gini Index, } \\
\text { Income } \\
\text { share }\end{array}$ & LE and IMR & $\begin{array}{l}\text { Income inequality not a } \\
\text { determinant of health }\end{array}$ \\
\hline $\mathrm{S} / \mathrm{N}$ & $\begin{array}{l}\text { Author(s), } \\
\text { Year }\end{array}$ & Sample & $\begin{array}{l}\text { Estimation } \\
\text { Method }\end{array}$ & $\begin{array}{l}\text { Proxy(s) } \\
\text { of Income } \\
\text { Inequality }\end{array}$ & $\begin{array}{l}\text { Proxy(s) of } \\
\text { Health }\end{array}$ & Findings \\
\hline 34. & $\begin{array}{l}\text { Karlsdotter et } \\
\text { al. (2012) }\end{array}$ & $\begin{array}{l}\text { Spanish life } \\
\text { conditions } \\
\text { survey for } \\
2007\end{array}$ & $\begin{array}{l}\text { Multilevel } \\
\text { logistic } \\
\text { regression }\end{array}$ & $\begin{array}{l}\text { Gini Index, } \\
\text { Theil and } \\
\text { Atkinson } \\
\text { indices }\end{array}$ & $\begin{array}{l}\text { SRH and } \\
\text { Chronic } \\
\text { illness }\end{array}$ & $\begin{array}{l}\text { Income inequality } \\
\text { measures had no } \\
\text { significant connection } \\
\text { with health outcomes }\end{array}$ \\
\hline 35. & $\begin{array}{l}\text { Feng et al. } \\
(2012)\end{array}$ & $\begin{array}{l}\text { Longitudinal } \\
\text { survey data } \\
\text { for } 23 \\
\text { Chinese } \\
\text { Provinces } \\
\text { (2008) }\end{array}$ & $\begin{array}{l}\text { Multilevel } \\
\text { logistic } \\
\text { model }\end{array}$ & Gini Index & SRH & $\begin{array}{l}\text { Province level income } \\
\text { inequality affects health } \\
\text { of elderly }\end{array}$ \\
\hline
\end{tabular}

Note: $\mathrm{AAMR} \Rightarrow$ Age Adjusted Mortality Rate; $\mathrm{ADL} \Rightarrow$ Activities of Daily Living; $\mathrm{BMI} \Rightarrow$ Body Mass Index; $\mathrm{CHC} \Rightarrow$ Chronic Health Conditions; CSMR $\Rightarrow$ Cause-Specific Mortality Rate; DCs $\Rightarrow$ Developed Countries; GI $\Rightarrow$ Gini Index; $\mathrm{HUI} \Rightarrow$ Health Utility Index; IMR $\Rightarrow$ Infant Mortality Rate; IRHI $\Rightarrow$ Income-Related Health Inequality; LDCs $\Rightarrow$ Less Developed Countries; LE $\Rightarrow$ Life Expectancy; MAMC $\Rightarrow$ Middle-upper Arm Muscle Circumference; SRH $\Rightarrow$ SelfRated Health; TB $\Rightarrow$ Tuberculosis; UMR $\Rightarrow$ Under-5 Mortality Rate; WHR $\Rightarrow$ Waist-to-Hip Ratio; WHZ $\Rightarrow$ Weight for Height-Z-score 


\begin{tabular}{|c|c|c|c|c|c|c|}
\hline $\mathrm{S} / \mathrm{N}$ & $\begin{array}{l}\text { Author(s), } \\
\text { Year }\end{array}$ & Sample & $\begin{array}{l}\text { Estimation } \\
\text { Method }\end{array}$ & $\begin{array}{l}\text { Proxy(s) } \\
\text { of Income } \\
\text { Inequality }\end{array}$ & $\begin{array}{l}\text { Proxy(s) of } \\
\text { Health }\end{array}$ & Findings \\
\hline 36. & Qi (2012) & $\begin{array}{l}57 \text { OECD \& } \\
\text { non-OECD } \\
\text { countries } \\
(2005)\end{array}$ & $\begin{array}{l}\text { Logistic } \\
\text { regression }\end{array}$ & Gini Index & $\mathrm{SRH}$ & $\begin{array}{l}\text { No independent adverse } \\
\text { effect of country income } \\
\text { inequality on SRH }\end{array}$ \\
\hline 37. & $\begin{array}{l}\text { Lau et al. } \\
(2012)\end{array}$ & $\begin{array}{l}\text { Hon Kong } \\
(1976-2000)\end{array}$ & $\begin{array}{l}\text { Negative } \\
\text { binomial } \\
\text { regression }\end{array}$ & Gini Index & $\begin{array}{l}\text { All- cause and } \\
\text { cause- } \\
\text { specific } \\
\text { deaths }\end{array}$ & $\begin{array}{l}\text { Positive association } \\
\text { between income } \\
\text { inequality and mortality }\end{array}$ \\
\hline 38. & $\begin{array}{l}\text { Grönqvist et } \\
\text { al. (2012) }\end{array}$ & $\begin{array}{l}\text { Swedish } \\
\text { population } \\
\text { aged 16-65 / } \\
16-74\end{array}$ & $\begin{array}{l}\text { OLS; } \\
\text { Instrumental } \\
\text { Variable }\end{array}$ & $\begin{array}{l}\text { Gini Index } \\
\text { and others }\end{array}$ & $\begin{array}{l}\text { Risk of being } \\
\text { hospitalised }\end{array}$ & $\begin{array}{l}\text { Income inequality has no } \\
\text { significant effect on } \\
\text { health }\end{array}$ \\
\hline 39. & $\begin{array}{l}\text { Sun et al. } \\
(2012)\end{array}$ & $\begin{array}{l}12,449 \\
\text { middle and } \\
\text { high schools' } \\
\text { students } \\
\text { from } 7 \\
\text { Chinese cities } \\
\text { (2002) }\end{array}$ & OLS & SPRII & $\begin{array}{l}\text { SRH, } \\
\text { depression, } \\
\text { stress and } \\
\text { cigarette } \\
\text { smoking }\end{array}$ & $\begin{array}{l}\text { Positive relationship } \\
\text { between SPRII and SRH } \\
\text { but no association } \\
\text { between SPRII and stress }\end{array}$ \\
\hline$S / N$ & $\begin{array}{l}\text { Author(s), } \\
\text { Year }\end{array}$ & Sample & $\begin{array}{l}\text { Estimation } \\
\text { Method }\end{array}$ & $\begin{array}{l}\text { Proxy(s) } \\
\text { of Income } \\
\text { Inequality }\end{array}$ & $\begin{array}{l}\text { Proxy(s) of } \\
\text { Health }\end{array}$ & Findings \\
\hline 40. & $\begin{array}{l}\text { Rasella et al. } \\
(2013)\end{array}$ & $\begin{array}{l}27 \text { Brazilian } \\
\text { states }(2000- \\
2009)\end{array}$ & $\begin{array}{l}\text { Fixed } \\
\text { Effects } \\
\text { Estimation }\end{array}$ & $\begin{array}{l}\text { Gini } \\
\text { Index/ } \\
\text { Percentile } \\
\text { dispersion } \\
\text { ratio }\end{array}$ & LE & $\begin{array}{l}\text { Income inequality } \\
\text { negatively associated } \\
\text { with life expectancy }\end{array}$ \\
\hline 41. & $\begin{array}{l}\text { Baeten et al. } \\
(2013)\end{array}$ & $\begin{array}{l}\text { China (1991- } \\
\text { 2006) }\end{array}$ & Probit Model & IRHI & $\mathrm{SRH}$ & $\begin{array}{l}\text { Rising income inequality } \\
\text { related to poor health }\end{array}$ \\
\hline 42. & $\begin{array}{l}\text { Pop et al. } \\
(2013)\end{array}$ & $\begin{array}{l}140 \text { countries } \\
(1987-2008)\end{array}$ & $\begin{array}{l}\text { Hybrid Fixed } \\
\text { Effects } \\
\text { Model }\end{array}$ & Net Gini & LE & $\begin{array}{l}\text { Higher inequality } \\
\text { associated with lower LE } \\
\text { for low- and middle- } \\
\text { income countries but } \\
\text { insignificant for DCs }\end{array}$ \\
\hline 43. & $\begin{array}{l}\text { Hamilton and } \\
\text { Kawachi } \\
(2013)\end{array}$ & $\begin{array}{l}35,620 \text { US } \\
\text { immigrants }\end{array}$ & $\begin{array}{l}\text { Logistic } \\
\text { regression }\end{array}$ & Gini Index & $\mathrm{SRH}$ & $\begin{array}{l}\text { Less income inequality } \\
\text { leads to better health }\end{array}$ \\
\hline
\end{tabular}

Note: $\mathrm{AAMR} \Rightarrow$ Age Adjusted Mortality Rate; $\mathrm{ADL} \Rightarrow$ Activities of Daily Living; $\mathrm{BMI} \Rightarrow$ Body Mass Index; $\mathrm{CHC} \Rightarrow$ Chronic Health Conditions; CSMR $\Rightarrow$ Cause-Specific Mortality Rate; DCs $\Rightarrow$ Developed Countries; GI $\Rightarrow$ Gini Index; $\mathrm{HUI} \Rightarrow$ Health Utility Index; IMR $\Rightarrow$ Infant Mortality Rate; IRHI $\Rightarrow$ Income-Related Health Inequality; LDCs $\Rightarrow$ Less Developed Countries; LE $\Rightarrow$ Life Expectancy; MAMC $\Rightarrow$ Middle-upper Arm Muscle Circumference; SRH $\Rightarrow$ SelfRated Health; TB $\Rightarrow$ Tuberculosis; UMR $\Rightarrow$ Under-5 Mortality Rate; WHR $\Rightarrow$ Waist-to-Hip Ratio; WHZ $\Rightarrow$ Weight for Height-Z-score 


\begin{tabular}{|c|c|c|c|c|c|c|}
\hline$S / N$ & $\begin{array}{l}\text { Author(s), } \\
\text { Year }\end{array}$ & Sample & $\begin{array}{l}\text { Estimation } \\
\text { Method }\end{array}$ & $\begin{array}{l}\text { Proxy(s) } \\
\text { of Income } \\
\text { Inequality }\end{array}$ & $\begin{array}{l}\text { Proxy(s) of } \\
\text { Health }\end{array}$ & Findings \\
\hline 44. & $\begin{array}{l}\text { Rajan et al. } \\
(2013)\end{array}$ & $\begin{array}{l}2001 \text { Indian } \\
\text { Census \& } \\
60 \text { th Round } \\
\text { of Indian } \\
\text { National } \\
\text { Statistical } \\
\text { Survey }\end{array}$ & $\begin{array}{l}\text { Logistic } \\
\text { regression }\end{array}$ & $\begin{array}{l}\text { Gini Index; } \\
90 / 10-\& \\
80 / 20- \\
\text { income } \\
\text { ratios }\end{array}$ & $\begin{array}{l}\text { IMR, UMR } \\
\text { and SRH }\end{array}$ & $\begin{array}{l}\text { Income inequality is a } \\
\text { strong determinant of } \\
\text { health at the individual } \\
\text { level but not at the state } \\
\text { and district level }\end{array}$ \\
\hline 45. & $\begin{array}{l}\text { Herzer and } \\
\text { Nunnenkamp } \\
(2014)\end{array}$ & $\begin{array}{l}19 \text { DCs and } \\
59 \text { LDCs }\end{array}$ & Panel ARDL & Gini Index & LE & $\begin{array}{l}\text { Income inequality } \\
\text { increases life expectancy } \\
\text { in DCs and significantly } \\
\text { reduces life expectancy in } \\
\text { LDCs }\end{array}$ \\
\hline 46. & $\begin{array}{l}\text { Rözer and } \\
\text { Volker (2015) }\end{array}$ & 30 DCs & $\begin{array}{l}\text { Multi-level } \\
\text { regression }\end{array}$ & Net Gini & $\mathrm{SRH}$ & $\begin{array}{l}\text { Income inequality had } \\
\text { negative impacts on } \\
\text { health, but the effect } \\
\text { faded away after age } 36\end{array}$ \\
\hline 47. & $\begin{array}{l}\text { Vincens and } \\
\text { Stafström } \\
(2015)\end{array}$ & $\begin{array}{l}\text { Brazil (2002- } \\
\text { 2009) }\end{array}$ & $\begin{array}{l}\text { Random } \\
\text { Effects }\end{array}$ & Gini Index & $\begin{array}{l}\text { Stroke } \\
\text { Mortality Rate }\end{array}$ & $\begin{array}{l}\text { Income inequality } \\
\text { independently associated } \\
\text { with stroke mortality } \\
\text { rates despite controlling } \\
\text { for GDP per capita }\end{array}$ \\
\hline$S / N$ & $\begin{array}{l}\text { Author(s), } \\
\text { Year }\end{array}$ & Sample & $\begin{array}{l}\text { Estimation } \\
\text { Method }\end{array}$ & $\begin{array}{l}\text { Proxy(s) } \\
\text { of Income } \\
\text { Inequality }\end{array}$ & $\begin{array}{l}\text { Proxy(s) of } \\
\text { Health }\end{array}$ & Findings \\
\hline 48. & $\begin{array}{l}\text { Siddigi et al. } \\
(2015)\end{array}$ & $\begin{array}{l}\text { US States } \\
(1990-2007)\end{array}$ & $\begin{array}{l}\text { Fixed } \\
\text { Effects and } \\
\text { Random } \\
\text { Effects }\end{array}$ & Gini & IMR & $\begin{array}{l}\text { Income inequality } \\
\text { inversely associated with } \\
\text { IMR, but with time, it had } \\
\text { increased positive } \\
\text { association with IMR }\end{array}$ \\
\hline 49. & $\begin{array}{l}\text { Bakkeli } \\
(2016)\end{array}$ & China & $\begin{array}{l}\text { Fixed } \\
\text { Effects }\end{array}$ & $\begin{array}{l}\text { Theil } \\
\text { index \& } \\
\text { Gini Index }\end{array}$ & $\begin{array}{l}\text { Blood } \\
\text { pressure, } \\
\text { WHR, MAMC } \\
\text { and } \\
\text { overweight }\end{array}$ & $\begin{array}{l}\text { Income inequality does } \\
\text { not have significant } \\
\text { impact on individual } \\
\text { health }\end{array}$ \\
\hline 50. & $\begin{array}{l}\text { Undurraga et } \\
\text { al. }(2016)\end{array}$ & $\begin{array}{l}\text { Bolivian } \\
\text { Amazon }\end{array}$ & $\begin{array}{l}\text { Negative } \\
\text { binomial } \\
\text { model, } \\
\text { ordered logit, } \\
\text { OLS and } \\
\text { logistic } \\
\text { regression }\end{array}$ & $\begin{array}{l}\text { Income } \\
\text { shares of } \\
\text { the } \\
\text { poorest } \\
20 \%\end{array}$ & $\begin{array}{l}\text { SRH, WHZ, } \\
\text { blood } \\
\text { pressure and } \\
\text { body fat }\end{array}$ & $\begin{array}{l}\text { Income transfers to rural } \\
\text { households increased } \\
\text { perceived stress of the } \\
\text { better off and reduced the } \\
\text { perceived health stress of } \\
\text { the poorest }\end{array}$ \\
\hline
\end{tabular}

Note: $\mathrm{AAMR} \Rightarrow$ Age Adjusted Mortality Rate; $\mathrm{ADL} \Rightarrow$ Activities of Daily Living; $\mathrm{BMI} \Rightarrow$ Body Mass Index; $\mathrm{CHC} \Rightarrow$ Chronic Health Conditions; CSMR $\Rightarrow$ Cause-Specific Mortality Rate; DCs $\Rightarrow$ Developed Countries; GI $\Rightarrow$ Gini Index; $\mathrm{HUI} \Rightarrow$ Health Utility Index; IMR $\Rightarrow$ Infant Mortality Rate; IRHI $\Rightarrow$ Income-Related Health Inequality; LDCs $\Rightarrow$ Less Developed Countries; LE $\Rightarrow$ Life Expectancy; MAMC $\Rightarrow$ Middle-upper Arm Muscle Circumference; SRH $\Rightarrow$ SelfRated Health; TB $\Rightarrow$ Tuberculosis; UMR $\Rightarrow$ Under-5 Mortality Rate; WHR $\Rightarrow$ Waist-to-Hip Ratio; WHZ $\Rightarrow$ Weight for Height-Z-score 


\begin{tabular}{|c|c|c|c|c|c|c|}
\hline$S / N$ & $\begin{array}{l}\text { Author(s), } \\
\text { Year }\end{array}$ & Sample & $\begin{array}{l}\text { Estimation } \\
\text { Method }\end{array}$ & $\begin{array}{l}\text { Proxy(s) } \\
\text { of Income } \\
\text { Inequality }\end{array}$ & $\begin{array}{l}\text { Proxy(s) of } \\
\text { Health }\end{array}$ & Findings \\
\hline 51. & $\begin{array}{l}\text { Elgar et al. } \\
(2016)\end{array}$ & $\begin{array}{l}40 \\
\text { industrialised } \\
\text { countries } \\
(1994-2014)\end{array}$ & $\begin{array}{l}\text { Hybrid FE } \\
\text { model }\end{array}$ & Net Gini & $\begin{array}{l}\text { Psychological } \\
\text { and physical } \\
\text { symptoms }\end{array}$ & $\begin{array}{l}\text { Early life income } \\
\text { inequality has adverse } \\
\text { impacts on health and } \\
\text { well-being of adolescent } \\
\text { girls }\end{array}$ \\
\hline 52. & $\begin{array}{l}\text { Ward \& Viner } \\
(2017)\end{array}$ & $\begin{array}{l}103 \text { countries } \\
(2012)\end{array}$ & $\begin{array}{l}\text { Cross- } \\
\text { Sectional } \\
\text { Regression }\end{array}$ & $\begin{array}{l}\text { National } \\
\text { Gini } \\
\text { Coefficient }\end{array}$ & IMR & $\begin{array}{l}\text { Gini positively associated } \\
\text { with increased all-cause } \\
\text { and communicable } \\
\text { disease mortality in both } \\
\text { sexes across all age } \\
\text { groups. }\end{array}$ \\
\hline 53. & $\begin{array}{l}\text { Ribeiro et al. } \\
(2017)\end{array}$ & $\begin{array}{l}\text { Meta- } \\
\text { analysis of } \\
27 \text { studies }\end{array}$ & $\begin{array}{l}\text { Meta- } \\
\text { regression }\end{array}$ & $\begin{array}{l}\text { Gini, Theil } \\
\& \\
\text { Atkinson } \\
\text { Indices }\end{array}$ & $\begin{array}{l}\text { Mental illness } \\
\text { related } \\
\text { morbidity }\end{array}$ & $\begin{array}{l}\text { Income inequality linked } \\
\text { with mental health } \\
\text { issues, with a small } \\
\text { overall effect }\end{array}$ \\
\hline 54. & $\begin{array}{l}\text { Pasqualini et } \\
\text { al. (2017) }\end{array}$ & $\begin{array}{l}12 \text { European } \\
\text { countries } \\
(1920-1956)\end{array}$ & OLS & Gini Index & $\begin{array}{l}\text { SRH, BMI, } \\
\text { chronic } \\
\text { diseases }\end{array}$ & $\begin{array}{l}\text { Country income } \\
\text { inequality are associated } \\
\text { negatively with health } \\
\text { conditions }\end{array}$ \\
\hline 55. & $\begin{array}{l}\text { Moeller et al. } \\
(2017)\end{array}$ & US & $\begin{array}{l}\text { Chi-square } \\
\text { tests }\end{array}$ & Gini Index & Oral health & $\begin{array}{l}\text { Income inequality affects } \\
\text { oral health }\end{array}$ \\
\hline$S / N$ & $\begin{array}{l}\text { Author(s), } \\
\text { Year }\end{array}$ & Sample & $\begin{array}{l}\text { Estimation } \\
\text { Method }\end{array}$ & $\begin{array}{l}\text { Proxy(s) } \\
\text { of Income } \\
\text { Inequality }\end{array}$ & $\begin{array}{l}\text { Proxy(s) of } \\
\text { Health }\end{array}$ & Findings \\
\hline 56. & $\begin{array}{l}\text { Odusanya } \\
\text { and Agboola } \\
(2017)\end{array}$ & Nigeria & ARDL & Gini Index & IMR & $\begin{array}{l}\text { Inequality exerts } \\
\text { negatively on health }\end{array}$ \\
\hline 57. & $\begin{array}{l}\text { Hill and } \\
\text { Jorgenson } \\
\text { (2018) }\end{array}$ & $\begin{array}{l}50 \text { US states } \\
\text { and } \\
\text { Columbia } \\
\text { District }(2000 \text {, } \\
\text { 2005, 2010) }\end{array}$ & $\begin{array}{l}\text { Two-way } \\
\text { Fixed } \\
\text { Effects }\end{array}$ & $\begin{array}{l}\text { Gl, income } \\
\text { shares for } \\
\text { top } 10,5 \& \\
1 \%\end{array}$ & LE & $\begin{array}{l}\text { State-level income } \\
\text { inequality inversely } \\
\text { associated with male and } \\
\text { female life expectancy. } \\
\text { Same general pattern } \\
\text { across all the measures } \\
\text { of income inequality. }\end{array}$ \\
\hline 58. & $\begin{array}{l}\text { Matthew and } \\
\text { Brodersen } \\
\text { (2018) }\end{array}$ & 50 US States & $\begin{array}{l}\text { Probit } \\
\text { regression }\end{array}$ & $\begin{array}{l}\text { State- } \\
\text { Level Gini } \\
\text { index }\end{array}$ & $\begin{array}{l}\text { Behavioural, } \\
\text { physical and } \\
\text { mental health }\end{array}$ & $\begin{array}{l}\text { Income inequality has } \\
\text { significant relationship } \\
\text { with all health outcomes }\end{array}$ \\
\hline 59. & $\begin{array}{l}\text { Vilda et al. } \\
\text { (2019) }\end{array}$ & $\begin{array}{l}\text { US Census } \\
\text { American } \\
\text { Community } \\
\text { Survey }\end{array}$ & $\begin{array}{l}\text { Poisson } \\
\text { Regression }\end{array}$ & Gini Coeff. & $\begin{array}{l}\text { Pregnancy } \\
\text { related } \\
\text { mortality }\end{array}$ & $\begin{array}{l}\text { Income-inequality is } \\
\text { related to pregnancy } \\
\text { related mortality }\end{array}$ \\
\hline
\end{tabular}

Note: $\mathrm{AAMR} \Rightarrow$ Age Adjusted Mortality Rate; $\mathrm{ADL} \Rightarrow$ Activities of Daily Living; $\mathrm{BMI} \Rightarrow$ Body Mass Index; $\mathrm{CHC} \Rightarrow$ Chronic Health Conditions; CSMR $\Rightarrow$ Cause-Specific Mortality Rate; DCs $\Rightarrow$ Developed Countries; GI $\Rightarrow$ Gini Index; $\mathrm{HUI} \Rightarrow$ Health Utility Index; IMR $\Rightarrow$ Infant Mortality Rate; IRHI $\Rightarrow$ Income-Related Health Inequality; LDCs $\Rightarrow$ Less Developed Countries; LE $\Rightarrow$ Life Expectancy; MAMC $\Rightarrow$ Middle-upper Arm Muscle Circumference; SRH $\Rightarrow$ SelfRated Health; TB $\Rightarrow$ Tuberculosis; UMR $\Rightarrow$ Under-5 Mortality Rate; WHR $\Rightarrow$ Waist-to-Hip Ratio; WHZ $\Rightarrow$ Weight for Height-Z-score 
pregnancies, women become depleted of resources that would otherwise be available for maintenance and repair of the body, and hence, lead to higher mortality. When child birth occurs at adolescent age, the chance for health complicacies increases which decreases life expectancy even more. edin represents education index. Educational attainment is one of the crucial social factors of increased life expectancy. Educated individuals tend to make better overall lifestyle choices which positively impacts the life longevity. heaexpen is the total health expenditure which is a determinant of human life quality and expectancy. Better health facilities lead to a healthier population. $u r b$ is the urban population, used as a measure of urbanisation. Urbanisation leads to multidirectional development, enhancement of lifestyle and increase in facilities including the health sectors which might improve quality of life. However, urbanisation also leads to environmental pollution, increase in crime activities amongst others which might prove counterproductive. regsc is the regime score, with lower scores being autocratic and higher scores being democratic form of government. It is a prevailing theory that democracy improves population health. Wigley and Akkoyunlu (2011) shed light on this argument by explaining that the democratic regimes distribute health-promoting resources more widely than autocratic regimes. saf is access to basic drinking water services. This is used as a control for basic sanitation. The world health organisation reports that every year more than 3.4 million people die as a result of water related diseases, making it the leading cause of disease and death around the world. Most of the victims are young children, the vast majority of whom die of illnesses caused by organisms that thrive in water sources contaminated by raw sewage. Hence, access to basic water services is an important determinant of life expectancy. The final control variable, $g d p p c$ is the real GDP per capita which is employed to control for economic prosperity. Economic prosperity leads to higher standard of life and subsequently, a healthier population.

The long-run relationship estimation implies that there exists a cointegrating relationship between the variables and that the series are non-stationarity. It is also to be taken into account that in longer panels, individual time series are probably affected by the same common factors, resulting in the presence of CSD. Hence, a couple of testing procedures are to be carried out preliminarily. I begin by testing for the presence of CSD following Pesaran (2015) and subsequently, test for the presence of unit roots. At first, the CIPS test developed by Pesaran (2007) is used for series with CSD. The CIPS test belongs to the second-generation panel unit root tests and is robust in presence of CSD. For testing the existence of cointegration, Kao cointegration test has been used and the lags were selected by AIC criterion.

The cointegration relation of equation (3) is estimated using Panel Dynamic OLS (PDOLS) estimator, the lags and leads of which were selected by AIC criterion and Fully Modified OLS estimator. By adding lags and leads to the variables in (3), these estimators control for potential simultaneity bias. The parametric DOLS is preferred to the nonparametric FMOLS in that the latter (unlike the former) imposes additional requirements that all variables should be integrated of the same order and that the regressors themselves should not be cointegrated. Hence, I will be mainly using PDOLS estimator. The FMOLS estimation outputs are provided just to observe whether there are any contradictory results. I, then estimate the PECM specification of equation (4) by using a standard fixed effects estimator.

\subsection{Instrument Core Identification Strategy}

Instrumenting inequality is called for because the outcome variable affects inequality in certain ways. It is an established fact that human capital is a necessary determinant of overall economic and individual well-being. Narrowing down on the health aspect of human capital, poor health can decline productivity by limiting one's ability to work, inhibiting educational attainment and hence, lead to medical debts and bankruptcy. This, in turn, thwarts income generation and aggravates income inequality situations. Evidences exist attesting to the direction of causality from health to income (for eg. Smith, 1999). Henceforth, I propose agricultural land per capita as an instrument for inequality. 
The single most crucial activity in Africa by far is agriculture. The agricultural sector provides employment to about two-thirds of the continent's working population and for each country, contributes an average of 30 to 60 percent of the GDP. Provided the fact that agriculture is the main livelihood of the people, agricultural land is most likely to be the key factor behind income generation, or consequently, the income inequality. A high correlation coefficient (0.3959) between the instrument and income inequality confirmed the potential explanatory power of the instrument. However, contrary to the argument that more agricultural land available to people would generate more income and reduce income inequality, the correlation was found positive. This is entirely plausible and the explanations are; firstly, the poorer population might own too less land to generate sufficient income. The entire land produce is used up for consumption by the owners. That brings me to the second point. Even if larger land areas are available, the poor land owners might not have enough means to produce at a sustainable level. In such cases, large land areas might become a burden. Thirdly, the data on agricultural land per capita might not capture the actual proportion of land ownerships. There exist uneven distributions of land owning to poor land governance and land reform policies. Hence, a well-off section of the population generates more income from the land while the conditions of the poor remain unaltered. Lastly, increasing population, disguised unemployment, absence of well-defined labour and agricultural laws and criminal activities like land grabbing leads to available agricultural land becoming a counterproductive factor for income inequality. There also exists empirical evidence that agricultural land can actually increase inequality (Nguyen, 2013).

The suitability of an instrument crucially depends on whether the instrument satisfies the exclusion restriction. The exclusion restriction for my identification strategy is satisfied if the instrument only affects life expectancy via income inequality and not directly. In my view, the condition holds firstly because agricultural land per capita has never been used as an explanatory variable for human capital formation or life expectancy. Secondly, in the events where agricultural land might lead to closely knit protected communities and enough productions for sustenance of life, life expectancy is likely to increase, which implies results that are biased in the direction opposite to the findings. Lastly, small land possessions are most likely to yield enough to impact life expectancy in itself. Additionally, almost no correlation (corr coefficient: -0.042) was found between the instrument and outcome variable which entrenched the suitability of the instrument.

\subsection{Data Type and Source}

The study involves annual data from a panel of 52 African countries, ranging over the period from 1995 to 2018 . All the data have been collected from World Bank World Development Indicators (2020 Q4 Edition), Polity5, UNFAO, UNDP HDR, SWIID and Penn World Table (version 9.1). The data for the regressions of historical context were collected from Clio Infra database.

\subsection{Results and Discussion}

The IV-2SLS estimation outputs are plotted in Table 2 along with the first stage regression and other necessary test results. 
Table 2

IV-2SLS Estimation

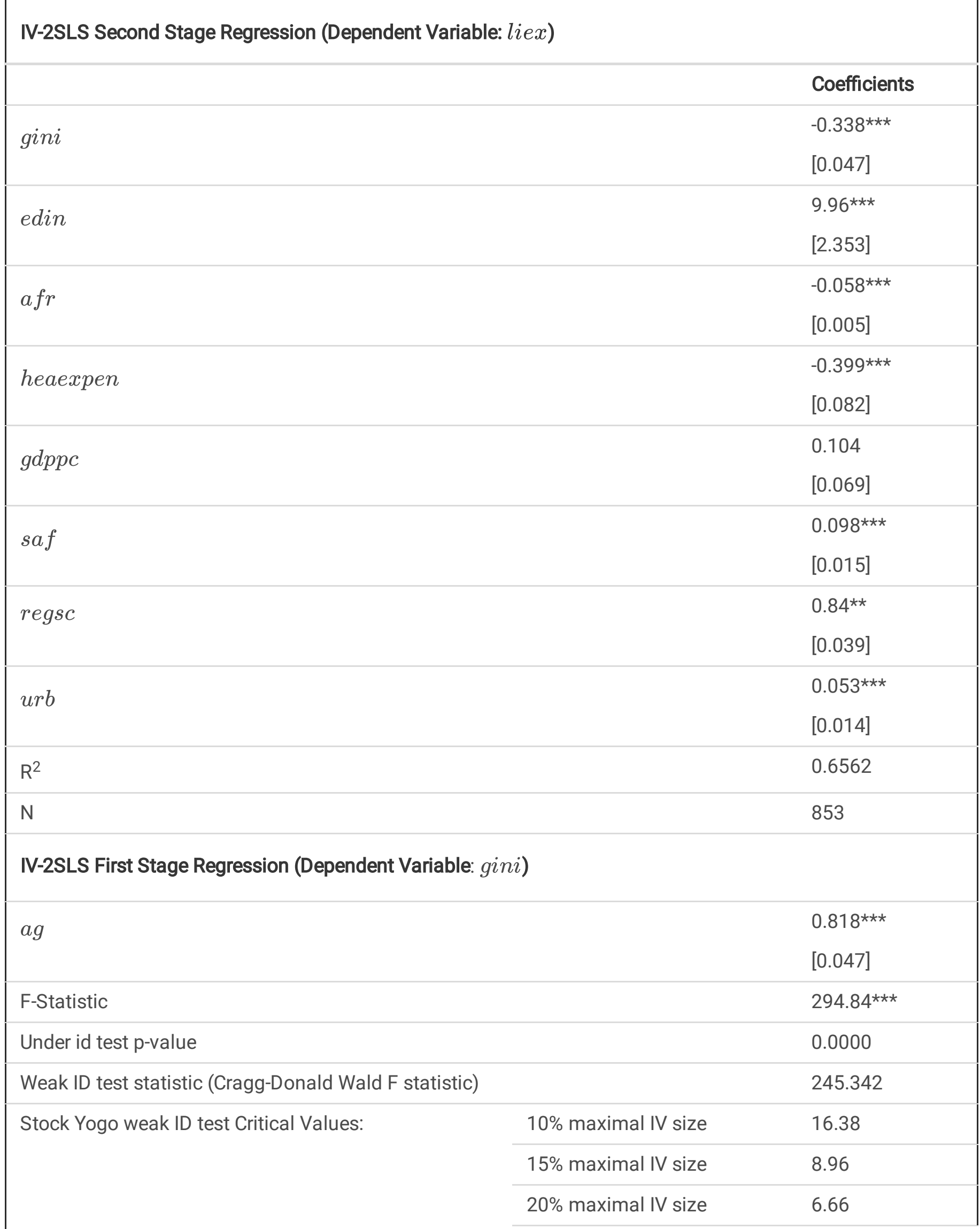

Note: $* \star \star, \star \star$ and $*$ represent significance at $1 \%, 5 \%$ and $10 \%$ level respectively. Standard errors are shown in [] 


\section{IV-2SLS Second Stage Regression (Dependent Variable: liex)}

$25 \%$ maximal IV size

5.53

Endog p-value

0.0103

Note: ${ }^{\star \star}, \star \star$ and * represent significance at $1 \%, 5 \%$ and $10 \%$ level respectively. Standard errors are shown in [] The IV 2SLS estimation outputs shows that, the coefficient of the gini variable is negative and significant at the $1 \%$ level which implies that increasing income inequality has a detrimental effect on life expectancy in the African population. All the outcomes are as par expectations except for health expenditure which has a significantly negative coefficient. Possible reasons could be inefficient spending, poor executions or non-optimal implementation of health schemes. The test results confirm the suitability of the instrument. The $F$ tests in the first stage regressions remain highly significant. The under-identification test statistic was found to be significant at $1 \%$ level implying no under identification. The weak identification test statistic is larger than all the stock-yogo weak id critical values implying no weak identification. Lastly, a significant endogeneity test statistic represents there is endogeneity.

The dynamic estimation analysis is initiated by interpreting the test results of Pesaran (2015) test for CSD. From the first panel of Table 3, the outputs show that all the series display significant CSD.

Table 3

CSD and Unit Root Test

\begin{tabular}{|c|c|c|c|c|c|c|c|c|c|}
\hline & \multicolumn{9}{|c|}{ Pesaran (2015) Test for Cross Sectional Dependence } \\
\hline & $\operatorname{liex}$ & gini & edin & $a f r$ & heaexpen & $g d p p c$ & saf & regsc & $u r b$ \\
\hline & $59.6^{\star \star \star}$ & $64.8^{\star \star \star}$ & $66.5^{\star \star \star}$ & $60.8^{\star \star \star}$ & $57.3^{\star \star \star}$ & $54.2^{\star \star \star}$ & $61.8^{\star \star \star}$ & $10.8^{\star \star \star}$ & $62.5^{\star \star \star}$ \\
\hline \multirow[t]{2}{*}{ lags } & \multicolumn{9}{|c|}{ Pesaran (2007) Panel Unit Root Test (CIPS) } \\
\hline & $\operatorname{liex}$ & gini & edin & $a f r$ & heaexpen & $g d p p c$ & saf & regsc & $u r b$ \\
\hline 0 & 4.66 & 5.05 & 1.07 & 8.41 & 0.27 & 1.87 & 1.82 & $-2.8^{\star \star \star}$ & 13.73 \\
\hline 1 & -0.91 & 5.03 & 2.23 & 0.34 & 2.64 & 1.09 & 5.6 & $-3.9^{\star \star \star}$ & $-2.3^{\star \star \star}$ \\
\hline
\end{tabular}

Following the CSD test, the unit root tests are done. The lag specification is ranged from 0 to 1 . The null hypothesis being existence of unit root, is accepted for all series with non-significant test statistics. Hence, the variables with significant test statistics (urb and regsc) are dropped out from the rest of the dynamic analysis.

The outcomes of the Kao cointegration tests (optimal lags selected by AIC criterion) are displayed in Table 4. Skimming through the test results, we see that three out of five test statistics are significant and hence, can confirm that there exists a long-run cointegration relationship in equation (3). 
Table 4

Kao Cointegration Test

\begin{tabular}{|c|c|}
\hline & Test Statistic \\
\hline Modified Dickey-Fuller t & 0.2694 \\
\hline Dickey-Fuller $\mathrm{t}$ & $-1.422 *$ \\
\hline Augmented Dickey-Fuller t & -0.4739 \\
\hline Unadjusted Modified Dickey-Fuller t & $4.051^{\star \star \star}$ \\
\hline Unadjusted Dickey-Fuller t & $1.6047 *$ \\
\hline \multicolumn{2}{|c|}{ Note: $\star \star \star, ~ * \star ~ a n d ~ *$ represent significance at $1 \%, 5 \%$ and $10 \%$ level respectively. } \\
\hline
\end{tabular}

Table 5

Inequality and Life Expectancy: The Long-Run Relationship

\begin{tabular}{|c|c|c|}
\hline \multicolumn{3}{|c|}{ Dependent Variable:liex } \\
\hline & PDOLS & FMOLS \\
\hline \multirow{2}{*}{ gini } & $-0.338 * \star \star *$ & $-0.213^{\star \star \star}$ \\
\hline & {$[0.068]$} & [0.021] \\
\hline \multirow{2}{*}{ edin } & $13.253^{\star \star \star}$ & $19.773^{\star \star \star}$ \\
\hline & {$[1.748]$} & {$[0.028]$} \\
\hline \multirow{2}{*}{$a f r$} & $-0.068^{\star \star \star}$ & $0.037^{*}$ \\
\hline & {$[0.007]$} & [0.021] \\
\hline \multirow{2}{*}{ heaexpen } & -0.001 & $0.204^{\star \star \star}$ \\
\hline & [0.035] & [0.038] \\
\hline \multirow{2}{*}{$g d p p c$} & $0.222^{\star \star \star}$ & 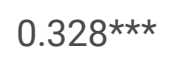 \\
\hline & {$[0.057]$} & [0.035] \\
\hline \multirow{2}{*}{ saf } & $0.318^{* \star *}$ & $0.352^{\star \star \star}$ \\
\hline & {$[0.011]$} & [0.032] \\
\hline Obs. (N) & 703 & 813 \\
\hline $\mathrm{R}^{2}$ & 0.9816 & 0.9743 \\
\hline
\end{tabular}

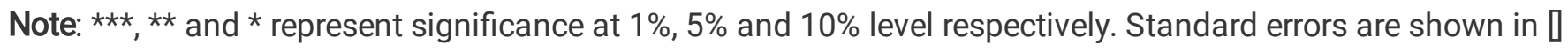
Estimation outcomes for (3) are shown in Table 5. The lags and leads for the PDOLS model are chosen as per AIC criterion. The model estimates a negative coefficient for income inequality and is highly significant at $1 \%$ level. The estimation output of the FMOLS model is mostly similar. Hence, a causal relationship could be established.

In Table 6, the estimation results of equation (4) are shown. $E C T$ is the error correction term. The result of a fixed effects model is plotted. 
Table 6

Income Inequality and Life Expectancy: PECM

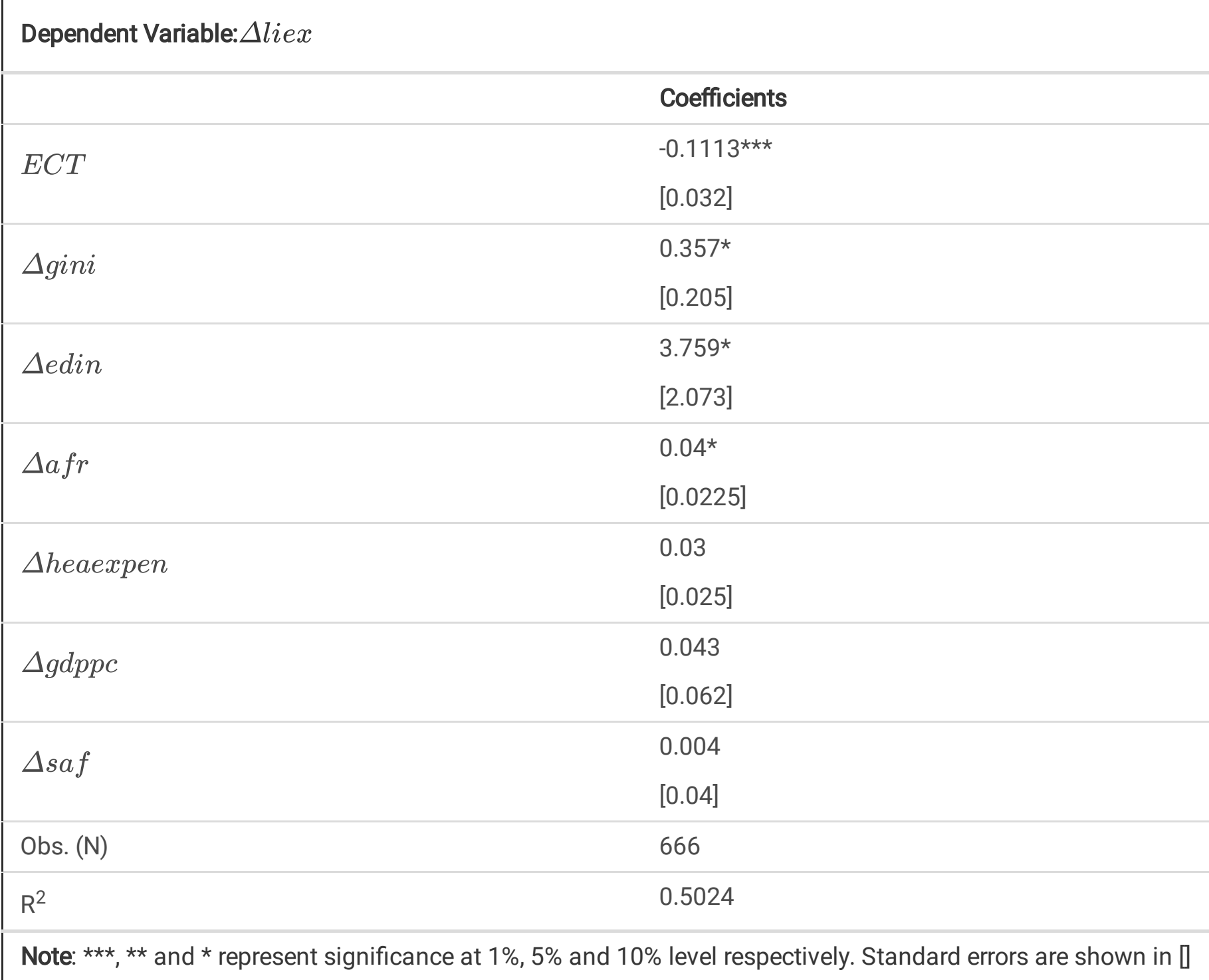

From the PECM outputs, it is observed that income inequality increased life expectancy in the short-run, which is a surprising result. A potential explanation for this outcome could be that in the short-run, the effects of the well-off section of the population is stronger. The coefficient of the ECT is negative and significant at the $1 \%$ level indicating that the short-run disturbances are persistent and that a deviation from the long-run equilibrium is corrected by approximately $11.13 \%$ over the following years. In other words, there is long-run convergence towards equilibrium.

\subsection{Robustness Check}

In order to check the robustness of the empirical findings, I resorted to fixed effects, random effects and pooled OLS estimators. The pooled OLS estimations were carried out with clustered standard errors by countries, given that standard errors are correlated among countries by construction. The outcomes are mostly similar (Appendix IV). Additionally, to have a brief historical context about the life expectancy-inequality relationship, data were collected between the period of 1820 and 1990, inclusive. However, the dataset was really thin due to lack of data availability. All the available variables and observations were included. No matter the inaccuracies arising due to less available control variables and data points, the results still confirm that income inequality has been inimical to life expectancy, keeping in mind the negative coefficients of the independent variable of interest (Appendix $\mathrm{V}$ ). 
The multiple-step checks confirmed the robustness of the empirical findings. Hence, we can safely establish the answer of the primary research question that inequality has negative impacts on human capital or precisely, life expectancy at birth in the African countries.

\section{Conclusion}

Comprehending the relevance and the impacts of diminishing socioeconomic inequalities, and in particular income inequality, in developing countries is of paramount importance. Inequality is detrimental not only from socioeconomic perspectives, but also for the health conditions of a population. Bearing the poor income inequality conditions in Africa and mediocre performance to ameliorate the condition in mind, this paper investigates the impacts of income inequality on the health aspect of human capital in the African continent.

The empirical analysis was carried out on a panel dataset consisting of data from 52 African nations, covering the period of 1995-2018. Life expectancy at birth has been used as measure for the population health. The relationship has been estimated using IV-2SLS estimation and a Panel Error Correction Model. Agricultural land per capita was used as the instrument for the Gini index. The long-run cointegrating relationship was estimated using PDOLS estimator. The outputs of both the static and dynamic estimation models suggested that income inequality has negatively affected life expectancy at birth. Though a positive short-run causal relationship was established, in the long-run, income inequality was prejudicial to population health. The robustness of the finding was proved following a series of steps.

However, the study is not free of shortcomings. Due to lack of data availability, the dataset had fewer observations and multiples gaps in cross sections. Hence, enhanced panel cointegration tests like Westerlund could not be performed. For the same reason, I could not perform dynamic CCE estimation which is robust in presence of cross-sectional dependence, even though cross-sectional dependence was confirmed. The data availability problem also led to dropping out multiple cross sections in the PDOLS estimation. Hence, there potentially exists certain degrees of inaccuracies in the dynamic analysis. Lastly, the dynamic model wasn't checked for endogeneity, or to be precise, the direction of causality. Further scopes on studies in this area includes finding more data, using more inclusive indices of income inequality, using household-level data for better policy formulation, checking the direction of causality and if there exists any reverse causality, more advanced estimation methods like CCE-GMM estimation should be employed finding suitable instruments.

\section{Declarations}

\section{Availability of data and materials}

The datasets generated and/or analysed during the current study are available in the World Bank World Development Indicators (2020 Q4 Edition), Polity5, UNFAO, UNDP HDR, SWIID, Penn World Table and Clio Infra repository,

\section{Competing interests}

The author declares that he has no competing interests.

\section{Funding}

Not Applicable

\section{Authors' contributions}

Not applicable (solo author) 


\section{Acknowledgement}

I would like to thank Prof. Dr. Joerg Baten for providing me his valuable insights in this article.

\section{References}

1. Aida J, Kondo K, Kondo N, Watt RG, Sheiham A, Tsakos G (2011) Income inequality, social capital and self-rated health and dental status in older Japanese. Soc Sci Med 73(10):1561-1568.

https://pubmed.ncbi.nlm.nih.gov/21982631/

2. Babones SJ (2008) Income inequality and population health: Correlation and causality. Soc Sci Med 66(7):16141626. https://doi.org/10.1016/j.socscimed.2007.12.012

3. Baeten S, Ourti TV, van Doorslaer E (2013) Rising inequalities in income and health in China: Who is left behind? Journal of Health Economics 32(6):1214-1229. http://dx.doi.org/10.1016/j.jhealeco.2013.10.00

4. Bakkeli NZ (2016) Income inequality and health in China: A panel data analysis. Soc Sci Med 157:39-47. http://dx.doi.org/10.1016/j.socscimed.2016.03.041

5. Baten J, Juif D (2014) A story of large landowners and math skills: Inequality and human capital formation in long-run development, 1820-2000. Journal of Comparative Economics 42(2):375-401. https://doi.org/10.1016/j.jce.2013.11.001

6. Biggs B, Kings L, Basu S, Stuckler D (2010) Is wealthier always healthier? The impact of national income level, inequality and poverty on public health in Latin America. Soc Sci Med 71(2):266-273. https://doi.org/10.1016/j.socscimed.2010.04.002

7. Birdsall N, Ross D, Sabot R (1995) Inequality and growth reconsidered: Lessons from East Asia. The World Bank Economic Review 9(3):477-508. https://www.jstor.org/stable/3989851

8. Chen Z, Meltzer D (2008) Beefing up with the Chans: Evidence for the effects of relative income and income inequality on health in China Health and Nutrition Survey. Soc Sci Med 66(11):2206-2217.

https://doi.org/10.1016/j.socscimed.2008.01.016

9. Chiang $T$ (1999) Economic transition and changing relation between income inequality and mortality in Taiwan: regression analysis. BMJ 319:1162-1165. https://doi.org/10.1136/bmj.319.7218.1162

10. Deaton A (2003) Health, Inequality, and Economic Development. JOURNAL OF ECONOMIC LITERATURE 41(1):113-158. https://www.aeaweb.org/articles?id=10.1257/002205103321544710

11. Detollenaere J, Desmarest AS, Boeckxstaens P, Willems S (2018) The link between income inequality and health in Europe, adding strength dimensions of primary care to the equation. Soc Sci Med 201:103-110. https://www.sciencedirect.com/science/article/pii/S0277953618300479

12. Eibner C, Evans WN (2005) Relative Deprivation, Poor Health Habits, and Mortality. The Journal of Juman Resources 40(3):591-620. http://jhr.uwpress.org/content/XL/3/591.abstract

13. Elgar FJ (2010) Income Inequality, Trust, and Population Health in 33 Countries. Am J Public Health 100(11):23112315. https://www.ncbi.nlm.nih.gov/pmc/articles/PMC2951926/

14. Elgar FJ, Gariépya G, Torsheim T, Currie C (2016) Early-life income inequality and adolescent health and well-being. Soc Sci Med 174:197-208. https://doi.org/10.1016/j.socscimed.2016.10.014

15. Esmaeili A, Mansouri S, Moshavash M (2011) Income inequality and population health in Islamic countries. Public Health 125(9):577-584. https://doi.org/10.1016/j.puhe.2011.06.003

16. Feng Z, Wang WW, Jones K, Li Y (2012) An exploratory multilevel analysis of income, income inequality and selfrated health of the elderly in China. Soc Sci Med 75(12):2481-2492. 
http://dx.doi.org/10.1016/j.socscimed.2012.09.028

17. Flegg AT (1979) Role of income inequality in the determination of birth rates. Population Studies 33(3):457-477. https://doi.org/10.2307/2173892

18. Flegg AT (1982) Inequality of income, illiteracy and medical care as determinants of infant mortality in underdeveloped countries. Population Studies 36(3):441-458. https://doi.org/10.1080/00324728.1982.10405597

19. Gravelle $\mathrm{H}$ (1998) How much of the relation between population mortality and unequal distribution of income is a statistical artefact? BMJ 316:382-385. https://doi.org/10.1136/bmj.316.7128.382

20. Gravelle H, Wildman J, Sutton MA (2002) Income, income inequality and health: what can we learn from aggregate data? Soc Sci Med 54(4):577-589. https://abdn.pure.elsevier.com/en/publications/income-income-inequality-andhealth-what-can-we-learn-from-aggreg

21. Grönqvist $H$, Johansson P, Niknami S (2012) Income inequality and health: Lessons from a refugee residential assignment program. Journal of Health Economics 31(4):617-629.

https://doi.org/10.1016/j.jhealeco.2012.05.003

22. Hales S, Howden-Chapman P, Salmond C, Woodward A, Mackenbach J (1999) National infant mortality rates in relation to gross national product and distribution of income. Lancet, 354(9195), 2047.

https://pubmed.ncbi.nlm.nih.gov/10636372/

23. Hamilton TG, Kawachi I (2013) Changes in income inequality and health of immigrants. Soc Sci Med 80:57-66. https://doi.org/10.1016/j.socscimed.2012.10.002

24. Herzer D, Nunnenkamp P (2014) Income Inequallty and health: Evidence from developed and developIng countrles. Diskussionspapierreihe Working Paper Series, 141, 1-57. http://www.economics-

ejournal.org/economics/journalarticles/2015-4/

25. Hill TD, Jorgenson A (2018) Bring out your dead!: A study of income inequality and life expectancy in the United States, 2000-2010. Health and Place, 49(November 2017), 1-6.

https://doi.org/10.1016/j.healthplace.2017.11.001

26. Judge K (1995) Income distribution and life expectancy: A critical appraisal. BMJ 311:1282-1287. https://doi.org/10.1136/bmj.311.7015.1282

27. Judge K, Mulligan J-A, Benzeval M (1998) Income inequality and population health. Soc Sci Med 46(4-5):567579. https://doi.org/10.1016/S0277-9536(97)00204-9

28. Kaplan GA, Pamuk ER, Lynch JW, Cohen RD, Balfour JL (1996) Inequality in income and mortality in the United States: analysis of mortality and potential pathways. BMJ 312:999-1003.

https://www.bmj.com/content/312/7037/999

29. Karlsdotter K, Martín JJM, del Amo González MP (2012) Multilevel analysis of income, income inequalities and health in Spain. Soc Sci Med 74(7):1099-1106. https://doi.org/10.1016/j.socscimed.2011.12.020

30. Kawachi I, Kennedy BP (1997) Health and social cohesion: why care about income inequality? BMJ 314(7086):1037-1040. https://pubmed.ncbi.nlm.nih.gov/9112854/

31. Kawachi I, Kennedy BP, Lochner K, Prothrow-Stitch D (1997) Social capital, income inequality, and mortality. Am J Public Health 87:1491-1498. https://doi.org/10.2105/AJPH.87.9.1491

32. Kondo N, Sembajwe G, Kawachi I, van Dam RM, Subramanian SV, Yamagata Z (2009) Income inequality mortality and self-rated health: meta-analysis of multilevel studies. BMJ 339:1178-1181.

https://doi.org/10.1136/bmj.b4471

33. Krugman PR (1996) The Spiral of Inequality. Mother Jones 21(6):44-49.

https://www.motherjones.com/politics/1996/11/spiral-inequality/

Page 21/26 
34. Lau EW, Schooling CM, Tin KY, Leung GM (2012) Income inequality and cause-specific mortality during economic development. Ann Epidemiol 22(4):285-294. http://dx.doi.org/10.1016/j.annepidem.2012.01.009

35. Layte R (2012) The Association Between Income Inequality and Mental Health: Testing Status Anxiety, Social Capital, and Neo-Materialist Explanations. Eur Sociol Rev 28(4):498-511. https://academic.oup.com/esr/articleabstract/28/4/498/431342? redirectedFrom=fulltext

36. Le Grand J (1987) Inequalities in health: some international comparisons. Eur Econ Rev 31:182-191. http://www.sciencedirect.com/science/article/pii/0014292187900304

37. LeClere FB, Soobadar M-J (2000) The effect of income inequality on the health of selected US demographic groups. Am J Public Health 90(12):1892-1897. https://doi.org/10.2105/AJPH.90.12.1892

38. Li H, Zhu Y (2006) Income, income inequality, and health: Evidence from China. Journal of Comparative Economics 34(4):668-693. https://doi.org/10.1016/j.jce.2006.08.005

39. Luo W, Xie Y (2020) Economic growth, income inequality and life expectancy in China. Social Science \& Medicine, 256. https://doi.org/10.1016/j.socscimed.2020.113046

40. Lynch JW, Smith GD, Kaplan GA, House JS (2000) Income inequality and mortality: importance to health of individual income, psychosocial environment, or material conditions. BMJ 320:1200-1204. https://pubmed.ncbi.nlm.nih.gov/10784551/

41. Matthew P, Brodersen DM (2018) Income inequality and health outcomes in the United States: An empirical analysis. The Social Science Journal 55(4):432-442. https://doi.org/10.1016/j.soscij.2018.05.001

42. McLeod CB, Lavis JN, Mustard CA, Stoddart GL (2003) Income inequality, household income, and health status in Canada: A prospective cohort study. Am J Public Health 93(8):1287-1293.

https://doi.org/10.2105/AJPH.93.8.1287

43. Mellor JM, Milyo J (2002) Income inequality and health status in the United States: Evidence from the current population survey. The Journal of Human Resources 37(3):510-539. https://doi.org/10.2307/3069680

44. Messias E (2003) Income inequality, illiteracy rate, and life expectancy in Brazil. Am J Public Health 93(8):12941296. https://doi.org/10.2105/AJPH.93.8.1294

45. Moeller J, Starkel R, Quiñonez C, Vujicic M (2017) Income inequality in the United States and its potential effect on oral health. The Journal of the American Dental Association 148(6):361-368.

http://dx.doi.org/10.1016/j.adaj.2017.02.052

46. Nguyen C (2013) The Impact of Agricultural Land on Poverty and Inequality in Rural Vietnam. MPRA Paper, 50478. https://mpra.ub.uni-muenchen.de/50478/

47. Odusanya IA, Agboola B (2017) Income, income inequality and health: Evidence from Nigeria. Izvestiya Journal of Varna University of Economics 61(4):345-361. http://journal.ue-

varna.bg/uploads/20180309062942_8481015625aa229d660273.pdf

48. Odusanya IA, Akinlo AE (2021) Income Inequality and Population Health in Sub-Saharan Africa: A Test of Income Inequality-Health Hypothesis. Journal of Population Social Studies 29:235-254. https://so03.tcithaijo.org/index.php/jpss/article/view/242032

49. Pampel FC, Pillai VK (1986) Patterns and determinants of infant mortality in developed nations, 1950-1975. Demography 23:525-542. https://doi.org/10.2307/2061349

50. Pasqualini M, Lanari D, Minelli L, Pieroni L, Salmasi L (2017) Health and income inequalities in Europe: What is the role of circumstances? Economics Human Biology 26:164-173. http://dx.doi.org/10.1016/j.ehb.2017.04.002

51. Pearce N, Smith GD (2003) Is Social Capital the Key to Inequalities in Health? Am J Publ Health 93(1):122-129. https://ajph.aphapublications.org/doi/10.2105/AJPH.93.1.122

Page 22/26 
52. Pei X, Rodriguez E (2006) Provincial income inequality and self-reported health status in China during 1991-7. J Epidemiol Community Health 60(12):1065-1069. https://www.ncbi.nlm.nih.gov/pmc/articles/PMC2465504/

53. Pesaran $\mathrm{MH}$ (2007) A simple panel unit root test in the presence of cross-section dependence. Journal of Applied Econometrics, 22(2), 265-312. https://onlinelibrary.wiley.com/doi/full/10.1002/jae.951

54. Pesaran MH (2015) Testing Weak Cross-Sectional Dependence in Large Panels. Econometric Reviews, 34(6-10), 1089-1117. https://www.tandfonline.com/doi/abs/10.1080/07474938.2014.956623

55. Pickett KE, Kelly S, Brunner E, Lobstein T, Wilkinson RG (2005) Wider income gaps, wider waistbands? An ecological study of obesity and income inequality. J Epidemiol Community Health 59(8):670-674. https://www.ncbi.nlm.nih.gov/pmc/articles/PMC1733121/

56. Pickett KE, Mookherjee J, Wilkinson RG (2005) Adolescent Birth Rates, Total Homicides, and Income Inequality In Rich Countries. Am J Public Health 95(7):1181-1183. https://www.ncbi.nlm.nih.gov/pmc/articles/PMC1449337/

57. Pickett KE, Wilkinson RG (2015) Income inequality and health: A causal review. Soc Sci Med 128:316-326. https://www.sciencedirect.com/science/article/pii/S0277953614008399

58. Pop IA, van Ingen E, van Oorschot W (2013) Inequality, wealth and health: Is decreasing inequality the key to create healthier societies? Soc Indic Res, 113, 1024-1043. https://link.springer.com/article/10.1007/s11205-012-0125-6

59. Qi Y (2012) The impact of income inequality on self-rated general health: Evidence from a cross-national study. Research in Social Stratification Mobility 30(4):451-471. https://doi.org/10.1016/j.rssm.2012.07.002

60. Rajan K, Kennedy J, King L (2013) Is wealthier always healthier in poor countries? The health implications of income, inequality, poverty, and literacy in India. Soc Sci Med 88:98-107.

http://dx.doi.org/10.1016/j.socscimed.2013.04.004

61. Ram R (2005) Income inequality, poverty, and population health: Evidence from recent data for the United States. Soc Sci Med 61(12):2568-2756. https://doi.org/10.1016/j.socscimed.2005.04.038

62. Ram R (2006) Further examination of the cross-country association between income inequality and population health. Soc Sci Med 62(3):779-791. https://doi.org/10.1016/j.socscimed.2005.06.034

63. Rasella D, Aquino R, Barreto ML (2013) Impact of income inequality on life expectancy in a highly unequal developing country: the case of Brazil. J Epidemiol Community Health 67(8):661-666. https://doi.org/10.1136/jech-2012-201426

64. Ribeiro WS, Bauer A, Andrade MCR, York-Smith M, Pan PM, Pingani L, Knapp M, Coutinho ESF, Evans-Lacko S (2017) Income inequality and mental illness-related morbidity and resilience: A systematic review and metaanalysis. The Lancet Psychiatry 4(7):554-562. http://dx.doi.org/10.1016/S2215-0366(17)30159-1

65. Rodgers GB (1979) Income and inequality as determinants of mortality: An international cross-section analysis. A Journal of Demography, 33(2), 343-351.

https://www.tandfonline.com/doi/abs/10.1080/00324728.1979.10410449

66. Rostila M, Kölegård ML, Fritzell J (2012) Income inequality and self-rated health in Stockholm, Sweden: A test of the 'income inequality hypothesis' on two levels of aggregation. Soc Sci Med 74(7):1091-1098.

https://www.sciencedirect.com/science/article/pii/S027795361200024X

67. Rözer JJ, Volker B (2015) Does income inequality have lasting effects on health and trust? Soc Sci Med 149:3745. https://doi.org/10.1016/j.socscimed.2015.11.047

68. Runciman W (1966) Relative Deprivation and Social Justice: A Study of Attributes to Social Inequality in Twentieth Century England. Routledge

69. Safaei J (2007) Income and health inequality across Canadian Provinces. Health Place 13(3):625-638. https://doi.org/10.1016/j.healthplace.2006.09.003

Page 23/26 
70. Shi L, Macinko J, Starfield B, Xu J, Politzer R (2003) Primary care, income inequality, and stroke mortality in the United States: A longitudinal analysis, 1985-1995. Stroke 34(8):1958-1964.

https://doi.org/10.1161/01.STR.0000082380.80444.A9

71. Shibuya K, Hashimoto H, Yano E (2002) Individual income, income distribution, and self-rated health in Japan: cross-sectional analysis of nationally representative sample. BMJ 324:16-19.

https://doi.org/10.1136/bmj.324.7328.16

72. Siddiqi A, Jones MK, Erwin PC (2015) Does higher income inequality adversely influence infant mortality rates? Reconciling descriptive patterns and recent research findings. Soc Sci Med 131:82-88.

http://dx.doi.org/10.1016/j.socscimed.2015.03.010

73. Smith GD (1996) Income inequality and mortality: why are they related? BMJ 312:987-988. https://www.bmj.com/content/312/7037/987

74. Smith JP (1999) Healthy Bodies and Thick Wallets: The Dual Relation between Health and Economic Status. Journal of Economic Perspectives 13(2):145-166. https://www.aeaweb.org/articles?id=10.1257/jep.13.2.145

75. Spencer N (2004) The effect of income inequality and macro-level social policy on infant mortality and low birthweight in developed countries-a preliminary systematic review. Child Care Health Dev 30(6):699-709. https://pubmed.ncbi.nlm.nih.gov/15527480/

76. Subramanian SV, Delgado I, Jadue L, Vega J, Kawachi I (2003) Income inequality and health: multilevel analysis of Chilean communities. J Epidemiol Community Health 57(11):844-846.

https://www.ncbi.nlm.nih.gov/pmc/articles/PMC1732331/

77. Subramanian SV, Kawachi I (2004) Income inequality and health: What have we learned so far? Epidemiologic Review 26(1):78-91. https://doi.org/10.1093/epirev/mxh003

78. Subramanian SV, Kawachi I, Smith GD (2007) Income inequality and the double burden of under-and overnutrition in India. Journal of Epidemiology Community Health 61(9):802-809. http://dx.doi.org/10.1136/jech.2006.053801

79. Sun P, Unger JB, Palmer P, Ma H, Xie B, Sussmen S, Johnson CA (2012) Relative income inequality and selected health outcomes in urban Chinese youth. Soc Sci Med 74(1):84-91.

https://doi.org/10.1016/j.socscimed.2011.10.010

80. Torre R, Myrskylä M (2011) Income inequality and population health: a panel data analysis on 21 developed countries. MPIDR Working Paper, 2011-006, 1-31. https://www.demogr.mpg.de/papers/working/wp-2011006.pdf

81. Truesdale BC, Jencks C (2016) The Health Effects of Income Inequality: Averages and Disparities. Annu Rev Public Health 37:413-430. https://doi.org/10.1146/annurev-publhealth-032315-021606

82. Undurraga EA, Behrman JR, Leonard WR, Godoy RA (2016) The effects of community income inequality on health: Evidence from a randomized control trial in the Bolivian Amazon. Soc Sci Med 149:66-75.

http://dx.doi.org/10.1016/j.socscimed.2015.12.003

83. Vilda D, Wallace M, Dyer L, Harville E, Theall K (2019) Income inequality and racial disparities in pregnancy-related mortality in the US. SSM-Population Health 9:100714. https://doi.org/10.1016/j.ssmph.2019.100477

84. Vincens N, Stafström M (2015) Income inequality, economic growth and stroke mortality in Brazil: Longitudinal and regional analysis 2002-2009. PLoS ONE 10(9):1-12. https://doi.org/10.1371/journal.pone.0137332

85. Vogli RD, Mistry R, Gnesott R, Cornia GA (2005) Has the relation between income inequality and life expectancy disappeared? Evidence from Italy and top industrialised countries. Journal of Epidemiol Community Health 59(2):158-162. https://pubmed.ncbi.nlm.nih.gov/15650149/

86. Walberg P, Mckee M, Shkolnikov V, Chenet L, Leon DA (1998) Economic change, crime, and mortality crisis in Russia: regional analysis. BMJ 317:312-318. https://doi.org/10.1136/bmj.317.7154.312

Page 24/26 
87. Ward JL, Viner RM (2017) The impact of income inequality and national wealth on child and adolescent mortality in low and middle-income countries. BMC Public Health 17(1):1-8. https://doi.org/10.1186/s12889-017-4310-z

88. Wen M, Browning CR, Cagney KA (2003) Poverty, affluence, and income inequality: neighborhood economic structure and its implications for health. Soc Sci Med 57(5):843-860. https://doi.org/10.1016/S02779536(02)00457-4

89. Wennemo I (1993) Infant mortality, public policy and inequality - a comparison of 18 industrialized countries 195085. Sociol Health Illn 15(4):429-446. https://doi.org/10.1111/j.1467-9566.1993.tb00354.x

90. Wigley S, Akkoyunlu A (2011) The Impact of Regime Type on Health: Does Redistribution Explain Everything? World Polit 63(4):647-677. https://pubmed.ncbi.nlm.nih.gov/22175088/

91. Wilkinson RG (1992) Income distribution and life expectancy. BMJ 304:165-168. https://doi.org/10.1136/bmj.304.6820.165

92. Wilkinson RG (1996) Unhealthy Societies The Afflictions of Inequality. Routledge, https://www.routledge.com/Unhealthy-Societies-The-Afflictions-of-Inequality/Wilkinson/p/book/9780415092357. https://www.routledge.com/Unhealthy-Societies-The-Afflictions-of-Inequality/Wilkinson/p/book/9780415092357

93. Wilkinson RG (1999) Income inequality, social cohesion, and health: clarifying the theory-a reply to Muntaner and Lynch. Int J Health Serv 29(3):525-543. https://pubmed.ncbi.nlm.nih.gov/10450545/

94. Wilkinson RG, Pickett KE (2006) Income inequality and population health: A review of and explanation of the evidence. Soc Sci Med 62(7):1768-1784. https://doi.org/10.1016/j.socscimed.2005.08.036

95. Wolfson MC, Kaplan G, Lynch J, Ross N, Backland E (1999) Relation between income inequality and mortality: Empirical demonstration. BMJ 319:953-955. https://doi.org/10.1136/bmj.319.7215.953

96. World Economic Forum (2011) Global Risks 2011. http://reports.weforum.org/global-risks-2011/

97. Wolfson, M. C., Kaplan, G., Lynch, J., Ross, N., \& Backland, E. (1999). Relation between income inequality and mortality: Empirical demonstration. British Medical Journal, 319, 953-955.

https://doi.org/10.1136/bmj.319.7215.953

98. World Economic Forum. (2011). Global Risks 2011. http://reports.weforum.org/global-risks-2011/

\section{Figures}




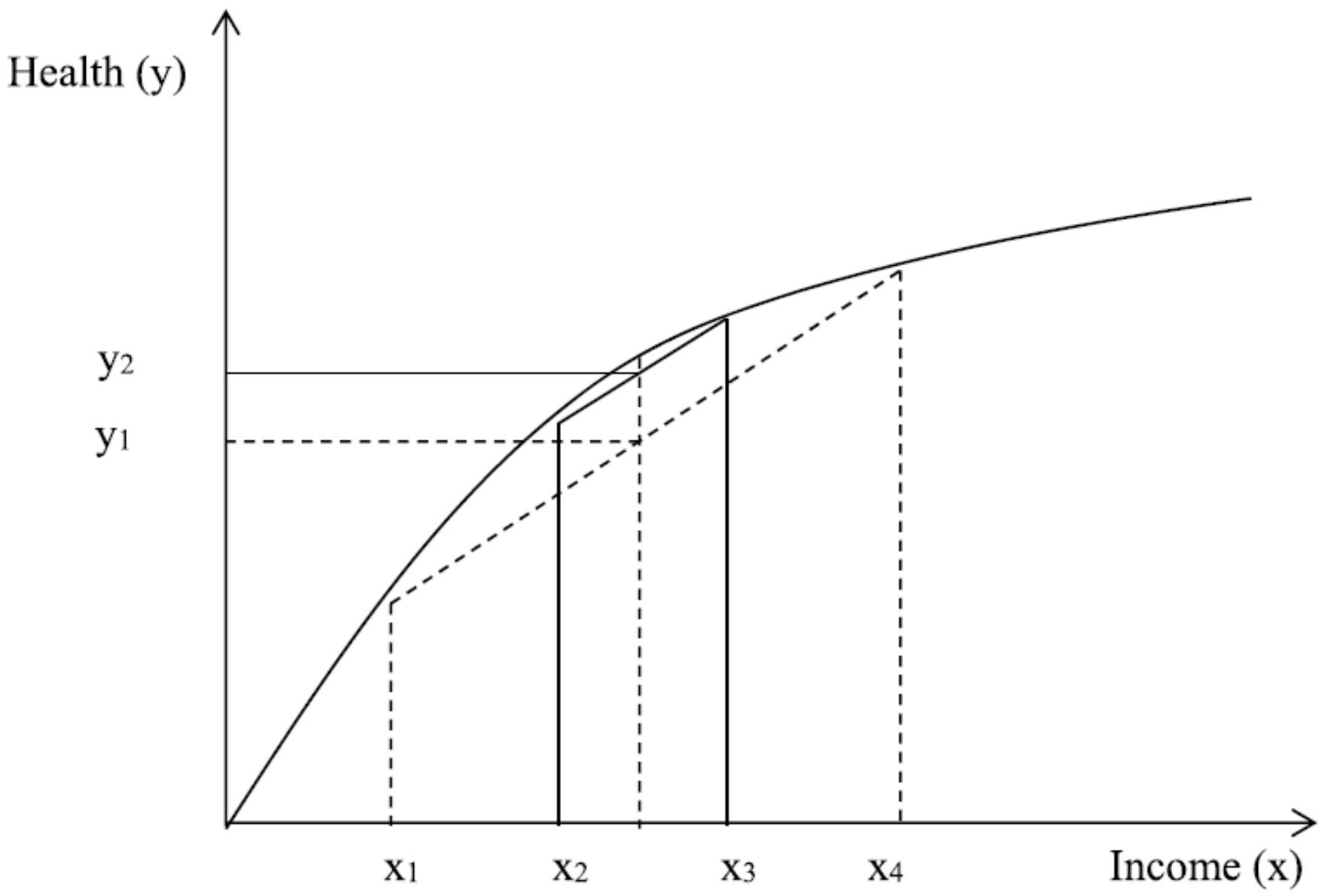

Figure 1

Individual-Level Relation between Income and Health

\section{Supplementary Files}

This is a list of supplementary files associated with this preprint. Click to download.

- APPENDIX.docX 